\title{
The hierarchy solution to the LHC inverse problem
}

\author{
James S. Gainer, ${ }^{a}$ Konstantin T. Matchev ${ }^{a}$ and Myeonghun Park ${ }^{b, c, d}$ \\ ${ }^{a}$ Physics Department, University of Florida, \\ Gainesville, FL 32611, U.S.A. \\ ${ }^{b}$ Asia Pacific Center for Theoretical Physics, \\ San 31, Hyoja-dong, Nam-gu, Pohang 790-784, Korea \\ ${ }^{c}$ Department of Physics, Postech, \\ Pohang 790-784, Korea \\ ${ }^{d}$ Kavli IPMU (WPI), The University of Tokyo, \\ Kashiwa, Chiba 277-8583, Japan \\ E-mail: jgainer@ufl.edu, matchev@phys.ufl.edu, parc@apctp.org
}

AbStract: Supersymmetric (SUSY) models, even those described by relatively few parameters, generically allow many possible SUSY particle (sparticle) mass hierarchies. As the sparticle mass hierarchy determines, to a great extent, the collider phenomenology of a model, the enumeration of these hierarchies is of the utmost importance. We therefore provide a readily generalizable procedure for determining the number of sparticle mass hierarchies in a given SUSY model. As an application, we analyze the gravity-mediated SUSY breaking scenario with various combinations of GUT-scale boundary conditions involving different levels of universality among the gaugino and scalar masses. For each of the eight considered models, we provide the complete list of forbidden hierarchies in a compact form. Our main result is that the complete (typically rather large) set of forbidden hierarchies among the eight sparticles considered in this analysis can be fully specified by just a few forbidden relations involving much smaller subsets of sparticles.

KeYWORDS: Supersymmetry Phenomenology

ARXiv EPrint: 1504.03689 


\section{Contents}

1 Introduction $\quad 2$

2 Notations and setup $\quad 8$

3 Linear algebraic approach $\quad 10$

$\begin{array}{lll}3.1 & \text { Theory } & 10\end{array}$

3.2 Specific examples 14

3.2.1 A forbidden hierarchy: $W Q$ in the CMSSM $(m=1) \quad 14$

3.2.2 A forbidden hierarchy: $L E U$ in the NUHM $(m=3) \quad 15$

3.2.3 An allowed hierarchy: $G B L$ in the NUHM $(m=3)$

4 Results: allowed $n$-particle (sub-)hierarchies $\quad 18$

4.1 The set of allowed hierarchies in the CMSSM 19

5 Results: the set of forbidden $n$-particle (sub-)hierarchies 22

5.1 Identifying all forbidden hierarchies in the CMSSM 25

5.2 Examples of forbidden hierarchies in the most general case $(m=8) \quad 28$

5.2.1 The mass hierarchy $G W Q B \quad 28$

5.2.2 The mass hierarchy WQGL 30

6 Conclusions and summary $\quad 31$

A Results on forbidden hierarchies $\quad 32$

A.1 Model $m=1$ (CMSSM) 32

A.2 Model $m=2 \quad 33$

A.3 Model $m=3$ (NUHM) 33

A.4 Model $m=4 \quad 34$

A.5 Model $m=5 \quad 34$

A.6 Model $m=6 \quad 34$

$\begin{array}{lll}\text { A.7 } & \text { Model } m=7 & 35\end{array}$

A.8 Model $m=8 \quad 35$

B Python package for studying hierarchies $\quad 35$

B.1 Data structures in Python 36

B.2 Hierarchy data 36

$\begin{array}{lll}\text { B.3 Methods } & 37\end{array}$

$\begin{array}{lll}\text { B.4 Using the code } & 38\end{array}$ 


\section{Introduction}

Low-energy supersymmetry (SUSY) [1] is a well-motivated paradigm for new physics beyond the Standard Model, as it explains the lightness of the recently-discovered Higgs boson [2, 3], provides a dark matter candidate [4], and, in some models, allows for the unification of gauge couplings, consistent with the low energy data [5]. That there is currently no compelling evidence of sparticle production at the CERN Large Hadron Collider (LHC) $[6,7]$ strongly motivates efforts to make sure that "no stone is left unturned" in searching for SUSY and/or setting more rigorous limits on the masses of sparticles. It is therefore important to understand all possible signatures of SUSY models; the rich phenomenology of SUSY means that there are unexplored "corners" of even well-known models like the CMSSM [8]. Such studies will become particularly valuable once a signal of new physics is observed, as they will help determine whether we are seeing a SUSY signal in the first place, and if so, what variety of SUSY model is responsible for it.

However, the exploration of SUSY collider phenomenology in full generality is a very challenging endeavor, complicated by the multitude of scenarios and the large number of SUSY parameters. Several approaches have been tried:

- Analysis of specific benchmark points [9-14]. The advantage of this approach is that it relies on well motivated and popular theory models. Benchmark points provide concrete, clear targets for discovery, and allow the sensitivities of different types of experiments to be compared in a meaningful way [15-17]. The downside is that the conclusions are very model-dependent and cannot be easily generalized to any arbitrary SUSY scenario.

- Analysis of the (phenomenologically relevant) MSSM parameter space (pMSSM) [1823]. The advantage here is that in principle one is exploring all corners of parameter space and encountering all phenomenologically interesting signatures. The disadvantage is that the large dimensionality of the pMSSM parameter space makes full coverage impossible. This has prompted studies in a correspondingly smaller parameter subspace, e.g., a nine parameter subset (pMSSM9) [24]. Alternatively, one could restrict one's attention to a specific sector of the model, e.g., the four lightest states in the new physics particle spectrum $[18,25,26]$. (See also the simplified model approach below.)

- Analysis of simplified models. The third, intermediate, approach is motivated by signature-based searches for new physics at the LHC. For any given experimental signature, one may consider only the particles that are relevant to this specific channel, arriving at a so-called "simplified model" with only a handful of parameters $[27,28]$. This approach inherits, to a degree, the advantages of the previous two methods, but the connection to the underlying high energy theory (and its fundamental parameters) is obscured.

The difficulty in exploring the phenomenology of the pMSSM parameter space in full generality manifests itself in the "LHC inverse problem" [29] of mapping an experimentally observed set of signatures at the LHC to a specific parameter point in theory space. The 
main challenge stems from the vastness of the SUSY parameter space - even under ideal circumstances the map is not unique and exhibits degeneracies. Furthermore, such maps so far have been constructed by (relatively sparse) scans in parameter space [30-33], and it is not clear to what extent the derived conclusions are robust and reliable.

A possible resolution to these problems was put forward in ref. [34], which proposed a more manageable and practical parameterization of theory space. The main idea is that the generic parameter space $\mathbb{R}^{n}$ of $n$ SUSY parameters can be thought of as the direct product of the set of all possible permutations $S_{n}$ of those $n$ parameters and the remaining coset $\mathbb{R}^{n} / S_{n}$ :

$$
\mathbb{R}^{n}=S_{n} \otimes \mathbb{R}^{n} / S_{n}
$$

The $n$ parameters considered in ref. [34] were all SUSY mass parameters, hence the permutations in $S_{n}$ were named "mass hierarchies", or "hierarchies" for short. The advantage of the decomposition (1.1) becomes evident when we consider the nature of a new physics signal at a collider and, in particular, the dependence on the two factors $S_{n}$ and $\mathbb{R}^{n} / S_{n}$. In general, the collider phenomenology of any new physics model depends on certain qualitative and quantitative aspects, where the former are parameterized by $S_{n}$, while the latter depend mostly on $\mathbb{R}^{n} / S_{n}$ :

- Quantitative aspects. Those are the factors which determine the overall signal rate, such as:

1. Signal production cross section. This depends on the magnitudes of the sparticle masses, which are encoded in the $\mathbb{R}^{n} / S_{n}$ factor alone. Generally, heavier particles have smaller cross-sections and vice versa.

2. Signal branching fractions. These are functions of the magnitudes of the mass splittings, which are also part of $\mathbb{R}^{n} / S_{n}$. For example, decay modes that are close to threshold, are kinematically suppressed, and the amount of suppression depends on exactly how close to threshold we are, i.e., on the size of the mass splitting.

3. Signal acceptances and efficiencies. These depend on the hardness of the SM decay products observed in the detector, which in turn is again a function of the magnitudes of the mass splittings. Parameter space points in $\mathbb{R}^{n} / S_{n}$ with large mass splittings lead to harder leptons, jets, etc., and correspondingly higher efficiencies. On the other hand, smaller mass splittings lead to degenerate scenarios with lower efficiencies, where discovery becomes problematic, see e.g. [35-37].

4. Relative contribution of strong versus electroweak production. In the years leading up to the LHC, it was usually assumed that strong SUSY production would dominate, and the first sign of SUSY would most likely be seen in squark and/or gluino production. Such expectations are based on the fact that strong production is enhanced due to the large strong coupling and the color multiplicity factors. However, this expectation is not true in models with heavy colored superpartners and light electroweak superpartners, where strong production is kinematically suppressed relative to electroweak production. The amount of 
suppression depends on the relative size of the superpartner masses, which is again parameterized by $\mathbb{R}^{n} / S_{n}$.

- Qualitative aspects. These are the factors that determine the type of discovery signature one is looking for, namely, the identity and multiplicity of SM particles in the final state. These qualitative features are mostly determined by the mass hierarchy, and can be parameterized by an element of the $S_{n}$ factor in (1.1).

The crucial observation of ref. [34] was that, as long as we are interested in the qualitative aspects of SUSY collider phenomenology, we are justified in focusing on $S_{n}$ only and studying hierarchies of sparticles without any reference to the actual sizes of their masses. Note that the number of elements in $S_{n}$ is a finite number (namely, $n !$ ), which allows a fully exhaustive exploration and classification of the sets of experimental signatures associated with each hierarchy. Somewhat surprisingly, such studies were able to reveal previously overlooked corners of pMSSM parameter space with dramatic, yet relatively unexplored, multi-lepton signatures [34]. While the analysis of [34] was originally applied only to pMSSM9, it was subsequently extended to include third generation sfermions and/or $R$-parity violation in the MSSM [38] and to the NMSSM [39].

In this paper we build on the work in $[34,38,39]$ and demonstrate the cataloguing of all possible hierarchies within specific SUSY breaking models. In the spirit of [34], we shall consider only the sparticles shown in table 1, namely, the gauginos and the first generation sfermions. We do not include any particles from the Higgs sector (Higgs bosons and higgsinos) for several reasons. First, the mass scale in the Higgs sector is set by a supersymmetry-preserving parameter, $\mu$, whose origin may be different from that of the other, supersymmetry breaking, mass parameters. Second, the running of the soft supersymmetry breaking Higgs mass parameters involves the Yukawa couplings, adding a great deal of complexity without a corresponding increase in insight. Similarly, we also do not include the third generation sfermions, because of the additional complications due to Yukawa and mixing effects. Both of those sectors can in principle be included; we plan to do so in a future study [40].

The mass spectrum will be parameterized by the parameters listed in the last row of table 1 . Some of these parameters correspond to several particles; for instance, the wino mass $M_{W}$ describes the mass of both a mostly wino-like neutralino $\tilde{w}^{0}$ and a mostly winolike chargino $\tilde{w}^{ \pm}$. (The remaining two gaugino mass parameters are denoted as $M_{B}$, the bino mass, and $M_{G}$, the gluino mass.) Likewise, we consider two degenerate generations of sfermions, so that each of the five sfermion masses $\left(M_{Q}, M_{U}, M_{D}, M_{L}\right.$ and $\left.M_{E}\right)$ describes particles in both the first and second generation. Finally, in the case of left-handed squarks (sleptons) the parameter $M_{Q}\left(M_{L}\right)$ refers to both members of the isodoublet, $\tilde{u}_{L}$ and $\tilde{d}_{L}$ $\left(\tilde{e}_{L}\right.$ and $\left.\tilde{\nu}_{L}\right)$. Tree-level sparticle mixing and one-loop corrections [41] complicate this story somewhat, since the experimentally measured mass eigenvalues are slightly different from the soft mass parameters in table 1. In what follows, we shall assume that the soft mass parameters

$$
\left\{M_{Q}, M_{U}, M_{D}, M_{L}, M_{E}, M_{B}, M_{W}, M_{G}\right\}
$$

have been extracted from the data via a global fit along the lines of refs. [42-45]. 


\begin{tabular}{|c|c|c|c|c|c|c|c|}
\hline$\tilde{u}_{L}, \tilde{d}_{L}$ & $\tilde{u}_{R}$ & $\tilde{d}_{R}$ & $\tilde{e}_{L}, \tilde{\nu}_{L}$ & $\tilde{e}_{R}$ & $\tilde{g}$ & $\tilde{w}^{ \pm}, \tilde{w}^{0}$ & $\tilde{b}^{0}$ \\
\hline$Q$ & $U$ & $D$ & $L$ & $E$ & $G$ & $W$ & $B$ \\
\hline 1 & 2 & 3 & 4 & 5 & 6 & 7 & 8 \\
\hline$M_{Q}$ & $M_{U}$ & $M_{D}$ & $M_{L}$ & $M_{E}$ & $M_{G}$ & $M_{W}$ & $M_{B}$ \\
\hline
\end{tabular}

Table 1. The set of SUSY particles considered in this analysis, shorthand notation (symbolic and numeric) for each multiplet, and the corresponding soft SUSY breaking mass parameter. Note our slightly unconventional notation for the three gaugino masses: $\left\{M_{B}, M_{W}, M_{G}\right\}$ instead of $\left\{M_{1}, M_{2}, M_{3}\right\}$ - in this paper the latter are reserved for the corresponding boundary conditions (1.5)-(1.7) at the GUT scale.

Given the eight experimentally measured mass parameters of table 1, the main question which we address in this paper is the following: can these measurements, by themselves, rule out specific SUSY-breaking scenarios? For our purposes here, a SUSY-breaking scenario is nothing more than a set of boundary conditions for the soft mass parameters of table 1 at some high energy scale. For concreteness, we shall illustrate our method with a SUGRAinspired scenario [46-49] in which the boundary conditions are specified at the GUT scale. We shall consider several popular variations of this SUGRA model, in which one imposes different sets of boundary conditions:

1. Scalar mass unification into $\mathrm{SU}(5)$ multiplets. Since the $\mathrm{SU}(5)$ is unbroken above the GUT scale $M_{\mathrm{GUT}}$, we shall always assume that squarks and sleptons belonging to the same $\mathrm{SU}(5)$ multiplet have a common mass at $M_{\mathrm{GUT}}$ :

$$
M_{Q}\left(M_{\mathrm{GUT}}\right)=M_{U}\left(M_{\mathrm{GUT}}\right)=M_{E}\left(M_{\mathrm{GUT}}\right) \equiv M_{10}, \quad M_{L}\left(M_{\mathrm{GUT}}\right)=M_{D}\left(M_{\mathrm{GUT}}\right) \equiv M_{5} .
$$

In general, $M_{5}$ and $M_{10}$ are independent input parameters - even if they start out equal at the Planck scale, they will be separated due to RGE running from the Planck scale down to the GUT scale $[50,51]$ or due to $D$-term contributions [52].

2. Scalar mass unification into $\mathrm{SO}(10)$ multiplets. A more constrained version of the model arises if we assume $\mathrm{SO}(10)$-like unification, where

$$
M_{5}=M_{10} \equiv M_{0} .
$$

3. Gaugino mass unification. In SU(5) GUTs, the gaugino masses may exhibit (some combination of) up to four ${ }^{1}$ different patterns $[53,54]$, so that in general

$$
\begin{aligned}
M_{B}\left(M_{\mathrm{GUT}}\right) & \equiv M_{1}, \\
M_{W}\left(M_{\mathrm{GUT}}\right) & \equiv M_{2}, \\
M_{G}\left(M_{\mathrm{GUT}}\right) & \equiv M_{3}
\end{aligned}
$$

\footnotetext{
${ }^{1}$ One for each irreducible representation appearing in the symmetric product $(24 \times 24)_{s}$ of two adjoints in $\mathrm{SU}(5)$.
} 
as free parameters at the GUT scale. We shall also optionally consider the usual assumption of gaugino unification,

$$
M_{1}=M_{2}=M_{3} \equiv M_{1 / 2} .
$$

4. Universal Higgs masses and third generation sfermions. Finally, although we are not explicitly considering the mass spectrum in the Higgs sector and the third generation sfermions, those mass parameters feed into the renormalization group equations (RGEs) through the hypercharge trace

$$
S \equiv \operatorname{Tr}\left(Y M^{2}\right) \equiv M_{H_{u}}^{2}-M_{H_{d}}^{2}+\sum_{i=1}^{3}\left(M_{Q_{i}}^{2}-2 M_{U_{i}}^{2}+M_{D_{i}}^{2}-M_{L_{i}}^{2}+M_{E_{i}}^{2}\right),
$$

where the index $i$ now runs over the three generations of sfermions. Extending (1.3) over the third generation as well, the last term in (1.9) is identically zero, and our last GUT scale assumption becomes

$$
S\left(M_{\mathrm{GUT}}\right)=0 \quad \Longleftrightarrow \quad M_{H_{u}}\left(M_{\mathrm{GUT}}\right)=M_{H_{d}}\left(M_{\mathrm{GUT}}\right)
$$

which is essentially the requirement of Higgs mass unification.

In this paper, we will always assume the GUT scale unification of sfermion families in $\mathrm{SU}(5)$ multiples described by eq. (1.3). By either imposing or not imposing the remaining three GUT scale assumptions (1.4), (1.8) and (1.10), we obtain a total of $2^{3}=8$ different model scenarios which are listed in table 2 . Each case has a different number of input mass parameters at the GUT scale (those parameters are listed explicitly in the last column of the table), so the sparticle spectrum is constrained to varying degrees in each scenario.

We proceed to study the allowed hierarchies in each model, using the shorthand notation from table 1 to label each hierarchy, ordering the particles from heaviest to lightest. For example, GQUDLWEB is a hierarchy with $M_{G}>M_{Q}>M_{U}>M_{D}>M_{L}>M_{W}>$ $M_{E}>M_{B}$. In all eight models from table 2, the number $d$ of input GUT-scale parameters (listed in the fifth column) is less than the number of measured parameters (1.2). Therefore, the GUT-scale boundary conditions will impose certain relationships among the low-energy parameters (1.2), and as a result, depending on the specific model, some hierarchies will be allowed, while others will be forbidden. The main purpose of this paper is to compile a complete catalogue of the allowed and forbidden hierarchies in each of the eight model scenarios from table 2. Our approach is completely general and can be easily applied to other SUSY-breaking mechanisms, where the soft mass parameters are given by a different set of boundary conditions at $M_{\mathrm{GUT}}$, or are generated at an initial scale different from $M_{\mathrm{GUT}}$. The method is not limited to SUSY models, and is equally applicable to non-SUSY scenarios, as long as the relevant mass parameters are evolved through a linear and homogeneous system of RGEs.

This paper is one in a long line of works addressing the major question of how to test for supersymmetry once a signal of new physics is seen [55]. After the initial determination of (some of) the SUSY mass parameters, one would like to know whether the data is 


\begin{tabular}{|c|c|c|c|c|c|}
\hline \multirow{2}{*}{$\begin{array}{c}\text { Case } \\
\text { number } \\
(m)\end{array}$} & \multicolumn{2}{|c|}{ Unification assumption } & Number & Input parameters \\
\cline { 2 - 4 } & Sfermion & Gaugino & Higgs & of inputs \\
(1.4) & $(1.8)$ & $(1.10)$ & $d^{(m)}$ & $\begin{array}{c}\text { at } M_{\mathrm{GUT}} \\
\vec{G}^{(m)}\end{array}$ \\
\hline 1 & yes & yes & yes & 2 & $M_{0}, M_{1 / 2}$ \\
2 & no & yes & yes & 3 & $M_{5}, M_{10}, M_{1 / 2}$ \\
3 & yes & yes & no & 3 & $M_{0}, M_{1 / 2}, S$ \\
4 & no & yes & no & 4 & $M_{5}, M_{10}, M_{1 / 2}, S$ \\
5 & yes & no & yes & 4 & $M_{0}, M_{1}, M_{2}, M_{3}$ \\
6 & no & no & yes & 5 & $M_{5}, M_{10}, M_{1}, M_{2}, M_{3}$ \\
7 & yes & no & no & 5 & $M_{0}, M_{1}, M_{2}, M_{3}, S$ \\
8 & no & no & no & 6 & $M_{5}, M_{10}, M_{1}, M_{2}, M_{3}, S$ \\
\hline
\end{tabular}

Table 2. The GUT scale assumptions behind each of the eight different SUGRA models studied in the paper. For completeness, we also list the number, $d$, of GUT scale input mass parameters in each case, as well as the names of those parameters.

consistent with supersymmetry in general, or with a specific model (e.g., one of the models in table 2). Several approaches are possible:

- Top-down approach. One can try to fit the data directly to the GUT-scale input parameters of the corresponding model [56-58]. In doing so, one is faced with the usual challenges of global minimization problems. In particular, one has to be careful to exhaustively cover all corners of parameter space, in order to be sure that a bad fit really excludes the model.

- Bottom-up approach. Alternatively, one can use the measured SUSY parameters at the electroweak scale as boundary conditions and run the RGEs in reverse back to the GUT scale $[59,60]$. This method provides a clear and intuitive picture of unification. However, since the RGE's are coupled, one needs a sufficiently large number of measurements in order to completely specify the initial conditions at low energies. Therefore, at the initial stages of the discovery, when only a fraction of the SUSY mass spectrum has been measured, this method generally does not apply.

- SUSY mass sum rules. The fact that the number of GUT scale inputs is less than the number of sparticle masses is reflected in the existence of certain model-dependent relations ("sum rules" for short) among the low energy parameters [61]. This idea has been richly explored in many different model scenarios [62-78], and has some relevance to our approach as well. For example, as illustrated below, we often find that the reason why certain mass hierarchies are not allowed is simply the fact that they violate one or more of the respective SUSY mass sum rules. However, our analysis will extend one step further and identify hierarchies which are consistent with the sum rules, but disallowed for other reasons - e.g., because they would 
require unphysical values for the GUT-scale boundary conditions or because they lead to tachyons in the spectrum.

The main advantages of our approach compared to these earlier studies are the following:

1. We often do not require knowledge of the complete mass spectrum in order to decide that a given model is ruled out. In the extreme cases, the knowledge of just two to four mass parameters can be already sufficient to discredit a given model hypothesis, regardless of the values of the remaining mass parameters (which may even be unmeasured).

2. We do not require any RGE analysis post-discovery, since all the required analytical work has already been performed ahead of time.

3. Our results are robust and reliable in the sense that they are obtained analytically and do not rely on any scanning of parameter spaces or on numerical fitting.

The paper is organized as follows. In section 2 we introduce our notations and describe the usual numerical procedure which relates GUT-scale to weak-scale parameters. The next sections describe various ways of determining if and understanding why a hierarchy is allowed or forbidden. In section 3 we explore a linear algebraic way to identify allowed and forbidden hierarchies. In section 4 we present our results on the allowed sets of mass hierarchies within each theory model scenario from table 2 . Then in section 5 we demonstrate how the enumeration of forbidden hierarchies is enormously simplified by the consideration of forbidden sub-hierarchies. In sections 4.1 and 5.1 we examine the use of mass sum rules to understand intuitively the sets of allowed and forbidden hierarchies, employing the CMSSM as a concrete example. Section 6 is reserved for our conclusions. In appendix A we summarize our results on the sets of forbidden hierarchies. The Python code which contains all the hierarchy information and which accompanies this paper is described in appendix B.

\section{Notations and setup}

The connection between the phenomenological parameters (1.2) measured at the electroweak scale $M_{\mathrm{EW}}$ and the corresponding input parameters (specified at the GUT scale $M_{\mathrm{GUT}}$ ) is provided by the MSSM RGEs, which allow exact analytical solutions at one loop. The starting point of our analysis is therefore the map [79]

$$
\left.\vec{W} \equiv\left(\begin{array}{c}
M_{Q}^{2} \\
M_{U}^{2} \\
M_{D}^{2} \\
M_{L}^{2} \\
M_{E}^{2} \\
M_{G}^{2} \\
M_{W}^{2} \\
M_{B}^{2}
\end{array}\right)_{M_{\mathrm{EW}}} \quad \equiv R^{(m)} \vec{G}^{(m)}\right|_{M_{\mathrm{GUT}}}
$$


where $\vec{W}$ is a vector of weak scale mass-squared parameters and $\vec{G}^{(m)}$ is the corresponding vector of independent GUT scale mass-squared parameters for case $m$ (see the last column in table 2). Note that the length of the vector $\vec{G}^{(m)}$ and the meaning of its components both depend on the specific SUSY scenario:

$$
\operatorname{dim}\left(\vec{G}^{(m)}\right)=d^{(m)}
$$

where the number $d$ of GUT scale input parameters is listed in the fifth column of table 2 . The matrix $R^{(m)}$, therefore, is an $8 \times d^{(m)}$ matrix which encodes the solution to the RGEs. It is instructive to illustrate eq. (2.1) with a couple of examples.

First, consider the most general case of model $m=8$, when there are $d^{(8)}=6$ independent inputs. The GUT scale parameter vector will then be ${ }^{2}$

$$
\vec{G}^{(8)}=\left(G_{1}^{(8)}, G_{2}^{(8)}, G_{3}^{(8)}, G_{4}^{(8)}, G_{5}^{(8)}, G_{6}^{(8)}\right)=\left(M_{10}^{2}, M_{5}^{2}, \tilde{S}, M_{3}^{2}, M_{2}^{2}, M_{1}^{2}\right),
$$

where as usual we have rescaled the hypercharge trace parameter as

$$
\tilde{S}=\frac{1}{66}\left(\frac{\alpha_{1}}{\alpha_{G}}-1\right) S .
$$

In what follows, the three $\alpha_{i} \equiv \frac{g_{i}^{2}}{4 \pi}$ parameterize the SM gauge couplings at the weak scale, while $\alpha_{G}$ stands for the corresponding unified gauge coupling at the GUT scale. In principle, the precise values of the gauge couplings depend on the scale of sparticle masses. For TeV scale SUSY, however, we shall use the typical values $\alpha_{G} \simeq 0.041, \alpha_{1} \simeq 0.017$, $\alpha_{2} \simeq 0.034$, and $\alpha_{3} \simeq 0.118$.

For model $m=8$, then, eq. (2.1) contains the $8 \times 6$ matrix $R^{(8)}$ :

$$
\vec{W} \equiv\left(\begin{array}{c}
M_{Q}^{2} \\
M_{U}^{2} \\
M_{D}^{2} \\
M_{L}^{2} \\
M_{E}^{2} \\
M_{G}^{2} \\
M_{W}^{2} \\
M_{B}^{2}
\end{array}\right)_{M_{\mathrm{EW}}}=\left(\begin{array}{cccccc}
1 & 0 & 1 & c_{3} & c_{2} & \frac{1}{36} c_{1} \\
1 & 0 & -4 & c_{3} & 0 & \frac{4}{9} c_{1} \\
0 & 1 & 2 & c_{3} & 0 & \frac{1}{9} c_{1} \\
0 & 1 & -3 & 0 & c_{2} & \frac{1}{4} c_{1} \\
1 & 0 & 6 & 0 & 0 & c_{1} \\
0 & 0 & 0 & a_{3} & 0 & 0 \\
0 & 0 & 0 & 0 & a_{2} & 0 \\
0 & 0 & 0 & 0 & 0 & a_{1}
\end{array}\right)\left(\begin{array}{c}
M_{10}^{2} \\
M_{5}^{2} \\
\tilde{S} \\
M_{3}^{2} \\
M_{2}^{2} \\
M_{1}^{2}
\end{array}\right)_{M_{\mathrm{GUT}}} \equiv R^{(8)} \vec{G}^{(8)}
$$

where the dimensionless coefficients $a_{i}$ and $c_{i}$ are defined as

$$
\begin{aligned}
a_{i} & \equiv \frac{\alpha_{i}^{2}}{\alpha_{G}^{2}}, \\
c_{i} & \equiv\left(\begin{array}{r}
\frac{2}{11} \\
\frac{3}{2} \\
-\frac{8}{9}
\end{array}\right)\left(1-a_{i}\right) .
\end{aligned}
$$

\footnotetext{
${ }^{2}$ Note that for the gaugino masses we continue to use the notation $M_{G}, M_{W}$ and $M_{B}$.
} 
Specifically, in our numerical analysis below we shall use the values $a_{1}=0.170, a_{2}=0.676$ and $a_{3}=8.29$. Analyses performed after a SUSY discovery could use updated values and scan over the then current experimental uncertainties on these parameters.

As another illustration, let us also discuss a simpler case - model $m=3$ has three input GUT scale parameters: $M_{0}, \tilde{S}$ and $M_{1 / 2}$. A non-zero value for $\tilde{S}$ at the GUT scale can be generated by non-universal Higgs masses, thus in what follows we shall label this model as NUHM $[80,81]$. The GUT scale parameter vector $\vec{G}^{(3)}$ can be taken to be

$$
\vec{G}^{(3)}=\left(G_{1}^{(3)}, G_{2}^{(3)}, G_{3}^{(3)}\right)=\left(M_{0}^{2}, \tilde{S}, M_{1 / 2}^{2}\right)
$$

and the corresponding $8 \times 3$ matrix $R^{(3)}$ will be

$$
R^{(3)}=\left(\begin{array}{rrr}
1 & 1 & c_{3}+c_{2}+\frac{1}{36} c_{1} \\
1 & -4 & c_{3}+\frac{4}{9} c_{1} \\
1 & 2 & c_{3}+\frac{1}{9} c_{1} \\
1 & -3 & c_{2}+\frac{1}{4} c_{1} \\
1 & 6 & c_{1} \\
0 & 0 & a_{3} \\
0 & 0 & a_{2} \\
0 & 0 & a_{1}
\end{array}\right)
$$

Finally, let us also mention the case of CMSSM, in which there are only two GUT scale parameters:

$$
\vec{G}^{(1)}=\left(G_{1}^{(1)}, G_{2}^{(1)}\right)=\left(M_{0}^{2}, M_{1 / 2}^{2}\right)
$$

and the corresponding $8 \times 2$ matrix $R^{(1)}$ is given by

$$
R^{(1)}=\left(\begin{array}{rr}
1 & c_{3}+c_{2}+\frac{1}{36} c_{1} \\
1 & c_{3}+\frac{4}{9} c_{1} \\
1 & c_{3}+\frac{1}{9} c_{1} \\
1 & c_{2}+\frac{1}{4} c_{1} \\
1 & c_{1} \\
0 & a_{3} \\
0 & a_{2} \\
0 & a_{1}
\end{array}\right)
$$

\section{Linear algebraic approach}

\subsection{Theory}

We now develop a criterion to decide whether, within a given model $m$ from table 2 , a specific $n$-particle hierarchy is possible or not. Note that in general we consider values for $n$ in the range

$$
2 \leq n \leq 8,
$$

i.e., we study not only full hierarchies of all 8 particles in eq. (1.2), but also arbitrary subsets of less than 8 particles $(n<8)$. As an extreme example, which we shall use in this 
section to illustrate the formal math, consider the 2-particle hierarchy $(n=2) W Q$ in the CMSSM model $(m=1)$.

Recall that the weak-scale masses-squared were arranged in the vector $\vec{W}$ introduced in (2.1). Thus we can specify a given (sub-)hierarchy of interest by either a string of $n$ letters taken from the second row of table 1 , or by a sequence of $n$ integers $\left(i_{1}, i_{2}, \ldots, i_{n}\right)$ denoting the corresponding components of the vector $\vec{W}$ and taken from the third row of table 1. For example, the hierarchy $M_{W}>M_{Q}$ can be represented by the letter string $W Q$ as explained in the introduction, or by the integer pair $\left(i_{1}, i_{2}\right)=(7,1)$ representing the respective components of the vector $\vec{W}$, namely $\left(W_{7}, W_{1}\right)$. Thus in general an $n$-particle hierarchy $\left(i_{1}, i_{2}, \ldots, i_{n}\right)$ is represented by an $n$-component vector

$$
\left(W_{i_{1}}, W_{i_{2}}, \cdots, W_{i_{n}}\right) .
$$

When we are dealing with a sub-hierarchy $(n<8)$, this vector has fewer than 8 components, and we find it convenient to always promote it back to an 8-component vector by adding the remaining $\vec{W}$ components $W_{j_{1}}, W_{j_{2}}, \cdots, W_{j_{8-n}}$ :

$$
\vec{H}=\left(W_{i_{1}}, W_{i_{2}}, \cdots, W_{i_{n}}, W_{j_{1}}, W_{j_{2}}, \cdots, W_{j_{(8-n)}}\right), \quad j_{k} \notin\left\{i_{1}, i_{2}, \cdots, i_{n}\right\} .
$$

In other words, the first $n$ components of $\vec{H}$ are the weak scale masses-squared in the $n$-particle hierarchy of interest (in decreasing order of mass), while the remaining $8-$ $n$ components are the remaining weak scale mass parameters, taken in arbitrary order. Operationally, we can build the vector $\vec{H}$ by multiplying $\vec{W}$ by the $8 \times 8$ square matrix

$$
U_{\vec{H}}=\left(\begin{array}{cccc}
\delta_{\left(i_{1}, 1\right)} & \delta_{\left(i_{1}, 2\right)} & \cdots & \delta_{\left(i_{1}, 8\right)} \\
\delta_{\left(i_{2}, 1\right)} & \delta_{\left(i_{2}, 2\right)} & \cdots & \delta_{\left(i_{2}, 8\right)} \\
\vdots & \vdots & \ddots & \vdots \\
\delta_{\left(i_{n}, 1\right)} & \delta_{\left(i_{n}, 2\right)} & \cdots & \delta_{\left(i_{n}, 8\right)} \\
\delta_{\left(j_{1}, 1\right)} & \delta_{\left(j_{2}, 2\right)} & \cdots & \delta_{(j 8,8)} \\
\vdots & \vdots & \ddots & \vdots \\
\delta_{\left(j_{(8-n)}, 1\right)} & \delta_{\left(j_{(8-n)}, 2\right)} & \cdots & \delta_{\left(j_{(8-n)}, 8\right)}
\end{array}\right)
$$

so that

$$
\vec{H} \equiv U_{\vec{H}} \vec{W}
$$

For our example of the 2-particle hierarchy $W Q=\left(W_{7}, W_{1}\right)$, we have $i_{1}=7$ and $i_{2}=1$. Then for concreteness we can take $j_{1}=2, j_{2}=3, j_{3}=4, j_{4}=5, j_{5}=6$ and $j_{6}=8$, so that eq. (3.5) reads

$$
\vec{H} \equiv\left(\begin{array}{l}
H_{1} \\
H_{2} \\
H_{3} \\
H_{4} \\
H_{5} \\
H_{6} \\
H_{7} \\
H_{8}
\end{array}\right)=\left(\begin{array}{llllllll}
0 & 0 & 0 & 0 & 0 & 0 & 1 & 0 \\
1 & 0 & 0 & 0 & 0 & 0 & 0 & 0 \\
0 & 1 & 0 & 0 & 0 & 0 & 0 & 0 \\
0 & 0 & 1 & 0 & 0 & 0 & 0 & 0 \\
0 & 0 & 0 & 1 & 0 & 0 & 0 & 0 \\
0 & 0 & 0 & 0 & 1 & 0 & 0 & 0 \\
0 & 0 & 0 & 0 & 0 & 1 & 0 & 0 \\
0 & 0 & 0 & 0 & 0 & 0 & 0 & 1
\end{array}\right)\left(\begin{array}{l}
W_{1} \\
W_{2} \\
W_{3} \\
W_{4} \\
W_{5} \\
W_{6} \\
W_{7} \\
W_{8}
\end{array}\right)=\left(\begin{array}{l}
W_{7} \\
W_{1} \\
W_{2} \\
W_{3} \\
W_{4} \\
W_{5} \\
W_{6} \\
W_{8}
\end{array}\right)
$$


Viable mass spectra exhibiting the required hierarchy are those for which the first $n$ components of the vector $\vec{H}$ are all positive and in decreasing order, while the remaining $8-$ $n$ components are just positive (and in any order). Since we are interested in a hierarchical ranking of the first $n$ components, it is convenient to define another vector, $\vec{\Delta}_{n}$, in terms of the mass-squared differences:

$$
\vec{\Delta}_{n} \equiv\left(\begin{array}{c}
H_{1}-H_{2} \\
H_{2}-H_{3} \\
\cdots \\
H_{(n-1)}-H_{n} \\
H_{n} \\
H_{n+1} \\
\cdots \\
H_{(8-n)}
\end{array}\right) \equiv D_{n} \vec{H}
$$

where the $8 \times 8$ square matrix $D_{n}$ is defined by

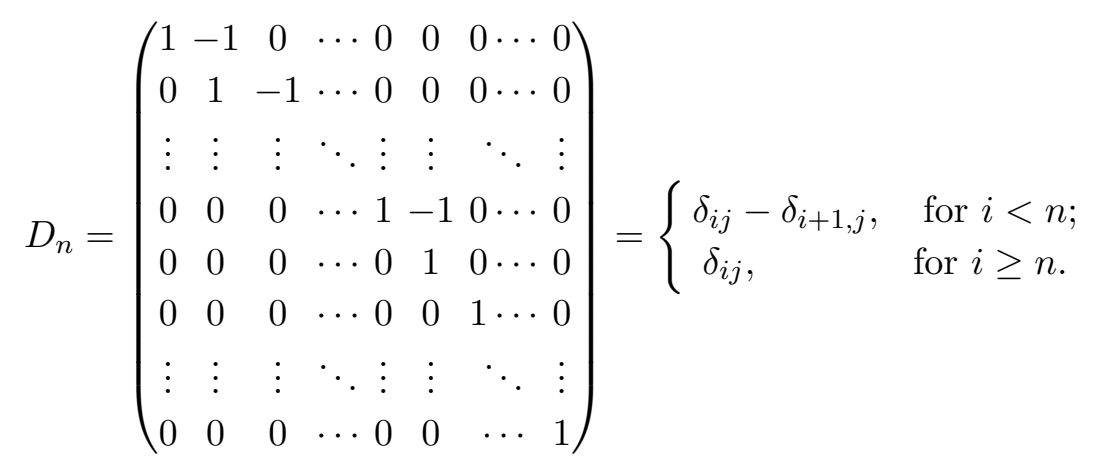

For the $W Q$ example in the CMSSM, the corresponding matrix $D_{2}$ is

$$
D_{2}=\left(\begin{array}{cccccccc}
1 & -1 & 0 & 0 & 0 & 0 & 0 & 0 \\
0 & 1 & 0 & 0 & 0 & 0 & 0 & 0 \\
0 & 0 & 1 & 0 & 0 & 0 & 0 & 0 \\
0 & 0 & 0 & 1 & 0 & 0 & 0 & 0 \\
0 & 0 & 0 & 0 & 1 & 0 & 0 & 0 \\
0 & 0 & 0 & 0 & 0 & 1 & 0 & 0 \\
0 & 0 & 0 & 0 & 0 & 0 & 1 & 0 \\
0 & 0 & 0 & 0 & 0 & 0 & 0 & 1
\end{array}\right)
$$

Combining (2.1), (3.5) and (3.7), we get

$$
\vec{\Delta}_{n, \vec{H}}^{(m)}=\left.D_{n} U_{\vec{H}} R^{(m)} \vec{G}^{(m)}\right|_{M_{\mathrm{GUT}}} .
$$

A set of GUT scale parameters $\vec{G}^{(m)}$ is able to produce a physically meaningful mass spectrum for the $n$-particle hierarchy under consideration ${ }^{3}$ if and only if all eight components

\footnotetext{
${ }^{3}$ Note that by construction $\vec{\Delta}_{n, \vec{H}}^{(m)}$ depends on the hierarchy, so that the vector $\vec{\Delta}_{n, \vec{H}}^{(m)}$ is defined on a case by case basis, as suggested by the index $\vec{H}$.
} 
of $\vec{\Delta}_{n, \vec{H}}^{(m)}$ are positive. We have thus reduced our original problem of proving the existence of the hierarchy (3.3) to the problem of finding solutions such that all components of $\vec{\Delta}_{n, \vec{H}}^{(m)}$ are positive.

The mathematical problem, therefore, is to determine whether for the $8 \times d^{(m)}$ matrix

$$
M_{n, \vec{H}}^{(m)}=D_{n} U_{\vec{H}} R^{(m)}
$$

appearing in (3.10), there exists $\vec{G}^{(m)} \in \mathbb{R}^{d^{(m)}}$ such that the vector

$$
\vec{\Delta}_{n, \vec{H}}^{(m)}=M_{n, \vec{H}}^{(m)} \vec{G}^{(m)}
$$

has only positive components. For this purpose, we can use a theorem known as Gordan's Alternative, [82] which states that for every $r \times s$ matrix, $A$, either

$$
\exists \vec{x} \in \mathbb{R}^{s} \text { such that } A \vec{x} \gg 0
$$

or

$$
\exists \vec{p} \in \mathbb{R}^{r} \text { such that } \vec{p}^{\top} A=0, \vec{p}>0 .
$$

Here we have used the usual mathematical notation that for a vector $\vec{a}, \vec{a} \gg 0$ indicates that all components of $\vec{a}$ are positive, while $\vec{a}>0$ only implies that all components are non-negative and in addition they cannot be all zero.

We can now directly make use of Gordan's alternative by identifying the matrix $A$ with our matrix $M_{n, \vec{H}}^{(m)}$ from (3.11). Then, if we can find a satisfactory solution to the equation

$$
\vec{p}^{\top} M_{n, \vec{H}}^{(m)}=0,
$$

the corresponding hierarchy is forbidden, i.e., there are no values of the GUT scale parameters $\vec{G}^{(m)}$ such that $\vec{\Delta}_{n, \vec{H}}^{(m)} \gg 0$. It is often easier to determine whether a solution to eq. (3.15) exists than to prove the existence of a vector $\vec{G}^{(m)}$ for which $M_{n, \vec{H}}^{(m)} \vec{G}^{(m)} \gg 0$. We shall demonstrate this with a few concrete examples below in section 3.2.

Note that this approach also allows us to add additional constraints on the range of the GUT scale parameters themselves. Since the GUT scale boundary conditions (the components of $\vec{G}^{(m)}$ ) are masses squared, one could demand that they are positive as well. This is certainly true for the gaugino masses squared $M_{i}^{2}$, and optionally for the scalar masses squared $M_{10}^{2}$ and $M_{5}^{2}$ [83]. The trace $\tilde{S}$ can, of course, have either sign.

If we demand that a particular GUT scale parameter $G_{i}^{(m)}$ be positive, we simply supplement the matrix $M_{n, \vec{H}}^{(m)}$ with a row vector $\vec{e}_{i}$ with components $\left(\vec{e}_{i}\right)_{j} \equiv \delta_{i j}$. Then the matrix $A$ becomes

$$
A=\left(\begin{array}{c}
M_{n, \vec{H}}^{(m)} \\
\vec{e}_{i}
\end{array}\right)
$$

Then,

$$
A \vec{G}^{(m)}=\left(\begin{array}{c}
M_{n, \vec{H}}^{(m)} \vec{G}^{(m)} \\
G_{i}^{(m)}
\end{array}\right)
$$


Gordan's Alternative tells us that either one can find acceptable ${ }^{4}$ values of the GUT scale inputs which will lead to the required hierarchy, or we can find a non-trivial solution to the matrix equation

$$
\vec{p}^{\top} A=\overrightarrow{0}
$$

such that all components of $\vec{p}$ are non-negative. Obviously, the procedure of enlarging the matrix $A$ as in (3.16) can be repeated for as many GUT scale parameters as necessary.

An alternative method, which may be easier to implement in practice, is to use the FindInstance [] command in Mathematica to obtain a list of all possible $n$-particle hierarchies in each of the eight SUSY scenarios. These lists are provided explicitly, along with methods to access this data, in the Python code described in appendix B.

\subsection{Specific examples}

\subsubsection{A forbidden hierarchy: $W Q$ in the CMSSM $(m=1)$}

Let us first apply the formalism of the preceding subsection to the case of the two-particle hierarchy $W Q$ in the CMSSM model $(m=1)$. The matrix $D_{2}$ was given in $(3.9)$, the matrix $U_{\vec{H}}$ was defined in (3.6), while $R^{(1)}$ was presented in (2.11). Thus from eq. (3.11) we can find the matrix $M_{2, \vec{H}}^{(1)}$ :

$$
M_{2, \vec{H}}^{(1)}=\left(\begin{array}{rc}
-1 & a_{2}-c_{3}-c_{2}-\frac{1}{36} c_{1} \\
1 & c_{3}+c_{2}+\frac{1}{36} c_{1} \\
1 & c_{3}+\frac{4}{9} c_{1} \\
1 & c_{3}+\frac{1}{9} c_{1} \\
1 & c_{2}+\frac{1}{4} c_{1} \\
1 & c_{1} \\
0 & a_{3} \\
0 & a_{1}
\end{array}\right) .
$$

The GUT scale parameters are given in (2.10). Requiring both to be positive, the enlarged matrix $A$ becomes

$$
A=\left(\begin{array}{rc}
-1 & a_{2}-c_{3}-c_{2}-\frac{1}{36} c_{1} \\
1 & c_{3}+c_{2}+\frac{1}{36} c_{1} \\
1 & c_{3}+\frac{4}{9} c_{1} \\
1 & c_{3}+\frac{1}{9} c_{1} \\
1 & c_{2}+\frac{1}{4} c_{1} \\
1 & c_{1} \\
0 & a_{3} \\
0 & a_{1} \\
1 & 0 \\
0 & 1
\end{array}\right)=\left(\begin{array}{rc}
-1 & -6.29 \\
1 & 6.97 \\
1 & 6.54 \\
1 & 6.49 \\
1 & 0.52 \\
1 & 0.15 \\
0 & 8.29 \\
0 & 0.17 \\
1 & 0 \\
0 & 1
\end{array}\right) .
$$

\footnotetext{
${ }^{4}$ Meaning $G_{i}^{(m)}>0$.
} 
Eqs. (3.18) now read

$$
\left(p_{1}, p_{2}, \ldots, p_{10}\right)\left(\begin{array}{rc}
-1 & -6.29 \\
1 & 6.97 \\
1 & 6.54 \\
1 & 6.49 \\
1 & 0.52 \\
1 & 0.15 \\
0 & 8.29 \\
0 & 0.17 \\
1 & 0 \\
0 & 1
\end{array}\right)=0 .
$$

Obviously,

$$
\vec{p}=\left(\begin{array}{c}
1 \\
0 \\
0 \\
0 \\
0 \\
0 \\
0 \\
0 \\
1 \\
6.29
\end{array}\right)
$$

is a solution to (3.21) and therefore, by Gordan's alternative, the hierarchy is not allowed. This can also be seen by noting that the first row of the $A$ matrix (3.20) contains only negative components, and the first equation in (3.12) reads

$$
\left(\Delta_{2, \vec{H}}^{(1)}\right)_{1}=-G_{1}^{(1)}-6.29 G_{2}^{(1)}=-M_{0}^{2}-6.29 M_{1 / 2}^{2} .
$$

It is clear that the right-hand side cannot be positive, as long as both $M_{0}^{2}$ and $M_{1 / 2}^{2}$ are positive as well.

\subsubsection{A forbidden hierarchy: $L E U$ in the NUHM $(m=3)$}

As a more complicated example of a forbidden hierarchy, consider the $L E U$ sub-hierarchy in the CMSSM with non-universal Higgs masses [80, 81] (which in our notation simply means that $\tilde{S}$ is no longer zero). For concreteness, we choose the remaining 5 particles to be $Q, D, G, W$, and $B$ and we form the extended vector $\vec{H}$ as in eq. (3.3):

$$
\vec{H}=\left(\begin{array}{c}
M_{L}^{2} \\
M_{E}^{2} \\
M_{U}^{2} \\
M_{Q}^{2} \\
M_{D}^{2} \\
M_{G}^{2} \\
M_{W}^{2} \\
M_{B}^{2}
\end{array}\right) .
$$


This defines the matrix

$$
U_{\vec{H}}=\left(\begin{array}{llllllll}
0 & 0 & 0 & 1 & 0 & 0 & 0 & 0 \\
0 & 0 & 0 & 0 & 1 & 0 & 0 & 0 \\
0 & 1 & 0 & 0 & 0 & 0 & 0 & 0 \\
1 & 0 & 0 & 0 & 0 & 0 & 0 & 0 \\
0 & 0 & 1 & 0 & 0 & 0 & 0 & 0 \\
0 & 0 & 0 & 0 & 0 & 1 & 0 & 0 \\
0 & 0 & 0 & 0 & 0 & 0 & 1 & 0 \\
0 & 0 & 0 & 0 & 0 & 0 & 0 & 1
\end{array}\right)
$$

Using (2.9), (3.8) and (3.25) we find the corresponding matrix $M_{3, \vec{H}}^{(3)}$ to be

$$
M_{3, \vec{H}}^{(3)}=\left(\begin{array}{rrr}
0 & -9 & c_{2}-\frac{3}{4} c_{1} \\
0 & 10 & -c_{3}+\frac{5}{9} c_{1} \\
1 & -4 & c_{3}+\frac{4}{9} c_{1} \\
1 & 1 & c_{3}+c_{2}+\frac{1}{36} c_{1} \\
1 & 2 & c_{3}+\frac{1}{9} c_{1} \\
0 & 0 & a_{3} \\
0 & 0 & a_{2} \\
0 & 0 & a_{1}
\end{array}\right) \simeq\left(\begin{array}{rrr}
0 & -9 & 0.37 \\
0 & 10 & -6.39 \\
1 & -4 & 6.54 \\
1 & 1 & 6.97 \\
1 & 2 & 6.49 \\
0 & 0 & 8.29 \\
0 & 0 & 0.68 \\
0 & 0 & 0.17
\end{array}\right) .
$$

In this model scenario, we only demand that $M_{0}^{2}$ and $M_{1 / 2}^{2}$ be positive, while $\tilde{S}$ can have either sign. Therefore, the matrix (3.26) is extended with only two extra rows:

$$
A=\left(\begin{array}{rrr}
0 & -9 & 0.37 \\
0 & 10 & -6.39 \\
1 & -4 & 6.54 \\
1 & 1 & 6.97 \\
1 & 2 & 6.49 \\
0 & 0 & 8.29 \\
0 & 0 & 0.68 \\
0 & 0 & 0.17 \\
1 & 0 & 0 \\
0 & 0 & 1
\end{array}\right)
$$

and the system of equations (3.18) to be studied is

$$
\left(p_{1}, p_{2}, \ldots, p_{10}\right)\left(\begin{array}{rrr}
0 & -9 & 0.37 \\
0 & 10 & -6.39 \\
1 & -4 & 6.54 \\
1 & 1 & 6.97 \\
1 & 2 & 6.49 \\
0 & 0 & 8.29 \\
0 & 0 & 0.68 \\
0 & 0 & 0.17 \\
1 & 0 & 0 \\
0 & 0 & 1
\end{array}\right)=0 .
$$


This system admits strictly positive non-trivial solutions, for example

$$
p_{2}=0.9 p_{1}, \quad p_{10}=\left(0.9 c_{3}-c_{2}+0.25 c_{1}\right) p_{1} \simeq 5.38 p_{1},
$$

for any positive value of $p_{1}$. According to Gordon's alternative, the existence of the solution (3.29) implies that the hierarchy $L E U$ is forbidden in the $m=3$ model. We shall see below that $L E U$ is indeed one of the 36 forbidden hierarchies in this model, see eq. (A.7).

\subsubsection{An allowed hierarchy: $G B L$ in the NUHM $(m=3)$}

We conclude this section with an example of an allowed hierarchy. Consider the subhierarchy $G B L$, which is one of the 198 allowed 3-particle hierarchies in model $m=3$ (cf. table 3). As in the previous subsection, in model $m=3$ the input parameters are still those given in (2.8), and the $R^{(3)}$ matrix is given by (2.9). For concreteness, we choose the remaining 5 particles to be $Q, U, D, E$, and $W$ and form the extended vector $\vec{H}$ as in eq. (3.3):

$$
\vec{H}=\left(\begin{array}{c}
M_{G}^{2} \\
M_{B}^{2} \\
M_{L}^{2} \\
M_{Q}^{2} \\
M_{U}^{2} \\
M_{D}^{2} \\
M_{E}^{2} \\
M_{W}^{2}
\end{array}\right)
$$

which defines the matrix

$$
U_{\vec{H}}=\left(\begin{array}{llllllll}
0 & 0 & 0 & 0 & 0 & 1 & 0 & 0 \\
0 & 0 & 0 & 0 & 0 & 0 & 0 & 1 \\
0 & 0 & 0 & 1 & 0 & 0 & 0 & 0 \\
1 & 0 & 0 & 0 & 0 & 0 & 0 & 0 \\
0 & 1 & 0 & 0 & 0 & 0 & 0 & 0 \\
0 & 0 & 1 & 0 & 0 & 0 & 0 & 0 \\
0 & 0 & 0 & 0 & 1 & 0 & 0 & 0 \\
0 & 0 & 0 & 0 & 0 & 0 & 1 & 0
\end{array}\right) .
$$

Then, using (2.9), (3.8), (3.31) and (3.11), we obtain the enlarged matrix (3.16)

$$
A=\left(\begin{array}{rrr}
0 & 0 & a_{3}-a_{1} \\
-1 & 3 & a_{1}-c_{2}-\frac{1}{4} c_{1} \\
1 & -3 & c_{2}+\frac{1}{4} c_{1} \\
1 & 1 & c_{3}+c_{2}+\frac{1}{36} c_{1} \\
1 & -4 & c_{3}+\frac{4}{9} c_{1} \\
1 & 2 & c_{3}+\frac{1}{9} c_{1} \\
1 & 6 & c_{1} \\
0 & 0 & a_{2} \\
1 & 0 & 0 \\
0 & 0 & 1
\end{array}\right) \simeq\left(\begin{array}{rrr}
0 & 0 & 8.12 \\
-1 & 3 & -0.35 \\
1 & -3 & 0.52 \\
1 & 1 & 6.97 \\
1 & -4 & 6.54 \\
1 & 2 & 6.49 \\
1 & 6 & 0.15 \\
0 & 0 & 0.68 \\
1 & 0 & 0 \\
0 & 0 & 1
\end{array}\right)
$$


The corresponding linear system of equations (3.18) is

$$
\left(\begin{array}{rrrrrrrrrr}
0 & -1 & 1 & 1 & 1 & 1 & 1 & 0 & 1 & 0 \\
0 & 3 & -3 & 1 & -4 & 2 & 6 & 0 & 0 & 0 \\
8.12 & -0.35 & 0.52 & 6.97 & 6.54 & 6.49 & 0.15 & 0.68 & 0 & 1
\end{array}\right)\left(\begin{array}{c}
p_{1} \\
p_{2} \\
\vdots \\
p_{10}
\end{array}\right)=0
$$

We want to show that this system has no solutions with $\vec{p}>0$. To easily see this, multiply the last equation by 4 and add to the first two equations, obtaining the equation

$$
\begin{aligned}
32.47 p_{1} & +0.58 p_{2}+0.09 p_{3}+29.87 p_{4}+23.18 p_{5} \\
& +28.97 p_{6}+7.60 p_{7}+2.70 p_{8}+p_{9}+4 p_{10}=0 .
\end{aligned}
$$

As all of the coefficients in eq. (3.34) are positive, there is clearly no valid solution for $\vec{p}$, and thus the hierarchy is allowed.

\section{Results: allowed $n$-particle (sub-)hierarchies}

For each of the eight model scenarios in table 2, only certain sets of hierarchies are allowed, in the sense that there exist values for the GUT scale parameters $\vec{G}^{(m)}$ which will give rise to the given mass ordering at the weak scale. Note that in searching for viable hierarchies, we are interested not only in obtaining the masses in the specified ordering, but in addition we require that all masses are physical, i.e., that all mass squared parameters at the weak scale (2.1) are positive. This is why the hierarchy vector $\vec{H}$ was defined in eq. (3.3) in terms of all eight components of the vector $\vec{W}$ from (2.1).

As indicated in (3.1), we shall also be interested in $n$-particle sub-hierarchies, i.e., sets of just a few particles with $n<8$. In doing so, we are motivated by the experimental reality - during the phase of initial discovery of supersymmetry, it is very likely that only a few superpartners will be seen. Therefore, at that stage it would make no sense to ask questions involving the unseen yet superpartners. Instead, one should focus on the question, given what has been observed so far, is the data consistent with a given theory model or not?

We shall therefore allow $n$ to vary within the full range (3.1). In general, for a given $n$, the number of all possible hierarchies which can be formed out of the set of eight observables (1.2) is given by $\frac{8 !}{(8-n) !}$ and listed in the last row of table 3 . The remaining rows of the table give the number of allowed n-particle hierarchies (in the sense described above), for each of the eight theoretical model scenarios from table 2.

Table 2 reveals a significant reduction in the number of possible hierarchies. Consider, for example, the least constrained model, Case 8, where there are as many as 6 input parameters at the GUT scale. Even then, out of the $8 !=40,380$ 8-particle permutations, only 7,766 hierarchies are possible (a little over 19\%). The other, more constrained models, exhibit a further reduction of the possible mass hierarchies. In the extreme case of the CMSSM (model $m=1$ in table 2), there are only nine 8-particle possibilities, as explained in section 4.1 below. 


\begin{tabular}{|c|r|r|r|r|r|r|r|}
\hline \multirow{2}{*}{$\begin{array}{c}\text { Case } \\
(m)\end{array}$} & \multicolumn{7}{|c|}{$n$} \\
\cline { 2 - 8 } & 2 & 3 & 4 & 5 & 6 & 7 & 8 \\
\hline 1 & 36 & 104 & 190 & 216 & 148 & 56 & 9 \\
\hline 2 & 42 & 154 & 382 & 604 & 570 & 290 & 61 \\
\hline 3 & 49 & 198 & 519 & 852 & 827 & 430 & 92 \\
\hline 4 & 51 & 240 & 757 & 1536 & 1887 & 1252 & 340 \\
\hline 5 & 55 & 312 & 1277 & 3232 & 4560 & 3254 & 913 \\
\hline 6 & 56 & 330 & 1521 & 4806 & 8684 & 7824 & 2699 \\
\hline 7 & 56 & 336 & 1591 & 5234 & 10240 & 10224 & 3940 \\
\hline 8 & 56 & 336 & 1648 & 6028 & 13778 & 16502 & 7766 \\
\hline $8 ! /(8-n) !$ & 56 & 336 & 1680 & 6720 & 20160 & 40320 & 40320 \\
\hline
\end{tabular}

Table 3. The number of allowed $n$-particle hierarchies for each of the eight models considered in table 2.

When we consider sub-hierarchies with $n<8$, the reduction in the number of allowed sub-hierarchies in table 3 is not as dramatic. This is because, when asking the question "Is this sub-hierarchy of $n$ particles allowed or not?" we allow the remaining (unseen) $8-n$ particles to have arbitrary masses. In particular, in models $m=7$ and $m=8$, all possible 2-particle and 3-particle hierarchies are still represented in the table, in spite of the significant reduction in terms of the number of allowed 8-particle hierarchies.

Of course, table 2 only tallies up the number of possible hierarchies in each model, but does not reveal which particular hierarchies are allowed. Given the large numbers seen in the table, giving here the complete list of all allowed hierarchies is impractical - the interested reader can easily generate the sets of allowed hierarchies from the accompanying code described in appendix B. This code can also answer queries about individual hierarchies (as well as sub-hierarchies) of interest.

Nevertheless, one may still wonder whether it is possible to somehow display the complete information about the sets of allowed hierarchies which are hiding behind table 3 . In the next section 5, we shall show that there is an elegant way of encoding and presenting the same amount of information. The key idea is, instead of studying the number of allowed hierarchies (which is typically very large, as seen in table 3), to focus on the (much smaller) number of forbidden hierarchies, starting with the smallest possible values of $n$.

\subsection{The set of allowed hierarchies in the CMSSM}

Table 2 revealed that in each of the eight model scenarios considered, the number of GUT scale input parameters $d^{(m)}$ is less than the number (eight) of weak scale parameters under consideration. Hence the hierarchies must satisfy $8-d^{(m)}$ sum rules, which explains the reduction in the number of allowed hierarchies observed in table 3.

We shall now illustrate how these sum rules help to identify allowed hierarchies using the example of the CMSSM $(m=1)$. In this case there are two input parameters $\left(M_{0}^{2}\right.$ and 
$M_{1 / 2}^{2}$ ), so we have the following six sum rules:

$$
\begin{aligned}
\left(5 c_{1}-12 c_{2}\right)\left(M_{D}^{2}-M_{U}^{2}\right)-4 c_{1}\left(M_{Q}^{2}-M_{U}^{2}\right) & =0, \\
3\left(5 c_{1}-12 c_{2}\right)\left(M_{L}^{2}-M_{U}^{2}\right)-\left(7 c_{1}-36 c_{2}+36 c_{3}\right)\left(M_{Q}^{2}-M_{U}^{2}\right) & =0, \\
3\left(5 c_{1}-12 c_{2}\right)\left(M_{E}^{2}-M_{U}^{2}\right)+\left(20 c_{1}-36 c_{3}\right)\left(M_{Q}^{2}-M_{U}^{2}\right) & =0, \\
\left(5 c_{1}-12 c_{2}\right) M_{G}^{2}+12 a_{3}\left(M_{Q}^{2}-M_{U}^{2}\right) & =0, \\
\left(5 c_{1}-12 c_{2}\right) M_{W}^{2}+12 a_{2}\left(M_{Q}^{2}-M_{U}^{2}\right) & =0, \\
\left(5 c_{1}-12 c_{2}\right) M_{B}^{2}+12 a_{1}\left(M_{Q}^{2}-M_{U}^{2}\right) & =0 .
\end{aligned}
$$

In obtaining these sum rules, we first used the first two rows of $R^{(1)}$ in (2.11) to express the GUT scale parameters, $M_{0}^{2}$ and $M_{1 / 2}^{2}$, in terms of the weak scale parameters, $M_{Q}^{2}$ and $M_{U}^{2}$,

$$
\begin{aligned}
M_{0}^{2} & =\frac{4\left(4 c_{1}+9 c_{3}\right)}{15 c_{1}-36 c_{2}} M_{Q}^{2}-\frac{c_{1}+36 c_{2}+36 c_{3}}{15 c_{1}-36 c_{2}} M_{U}^{2}, \\
M_{1 / 2}^{2} & =\frac{12}{5 c_{1}-12 c_{2}}\left(M_{U}^{2}-M_{Q}^{2}\right) .
\end{aligned}
$$

Then, the remaining six rows of the matrix $R^{(1)}$ result in the sum rules (4.1)-(4.6).

We note that the overall mass scale does not have any impact on whether a given hierarchy is allowed or not. We can therefore remove one degree of freedom; here we choose this degree of freedom to be $M_{Q}^{2}$. Since the CMSSM has only two input parameters (i.e., $d^{(1)}=2$ ), removing the overall scale as $M_{Q}^{2}$ leaves us with only one relevant degree of freedom, which we can take to be the ratio $\frac{M_{U}^{2}}{M_{Q}^{2}}$. The sum rules (4.1)-(4.6) can then be rewritten as

$$
\begin{aligned}
& \frac{M_{D}^{2}}{M_{Q}^{2}}=\frac{c_{1}-12 c_{2}}{5 c_{1}-12 c_{2}}\left(\frac{M_{U}^{2}}{M_{Q}^{2}}\right)+\frac{4 c_{1}}{5 c_{1}-12 c_{2}} \\
& \frac{M_{L}^{2}}{M_{Q}^{2}}=\frac{8 c_{1}-36 c_{3}}{15 c_{1}-36 c_{2}}\left(\frac{M_{U}^{2}}{M_{Q}^{2}}\right)+\frac{7 c_{1}-36 c_{2}+36 c_{3}}{15 c_{1}-36 c_{2}} \\
& \frac{M_{E}^{2}}{M_{Q}^{2}}=\frac{35 c_{1}-36 c_{2}-36 c_{3}}{15 c_{1}-36 c_{2}}\left(\frac{M_{U}^{2}}{M_{Q}^{2}}\right)-\frac{20 c_{1}-36 c_{3}}{15 c_{1}-36 c_{2}} \\
& \frac{M_{G}^{2}}{M_{Q}^{2}}=\frac{12 a_{3}}{5 c_{1}-12 c_{2}}\left(\frac{M_{U}^{2}}{M_{Q}^{2}}\right)-\frac{12 a_{3}}{5 c_{1}-12 c_{2}} \\
& \frac{M_{W}^{2}}{M_{Q}^{2}}=\frac{12 a_{2}}{5 c_{1}-12 c_{2}}\left(\frac{M_{U}^{2}}{M_{Q}^{2}}\right)-\frac{12 a_{2}}{5 c_{1}-12 c_{2}} \\
& \frac{M_{B}^{2}}{M_{Q}^{2}}=\frac{12 a_{1}}{5 c_{1}-12 c_{2}}\left(\frac{M_{U}^{2}}{M_{Q}^{2}}\right)-\frac{12 a_{1}}{5 c_{1}-12 c_{2}}
\end{aligned}
$$

which are clearly linear equations in $\frac{M_{U}^{2}}{M_{Q}^{2}}$. In general, the ratio $\frac{M_{U}^{2}}{M_{Q}^{2}}$ can take values in the interval $(0, \infty)$. However, not all values will lead to physically viable mass spectra, as illustrated in figure 1. In particular, some of the weak scale masses squared can become negative and lead to tachyonic particles. As observed in in figure 1, the most stringent 


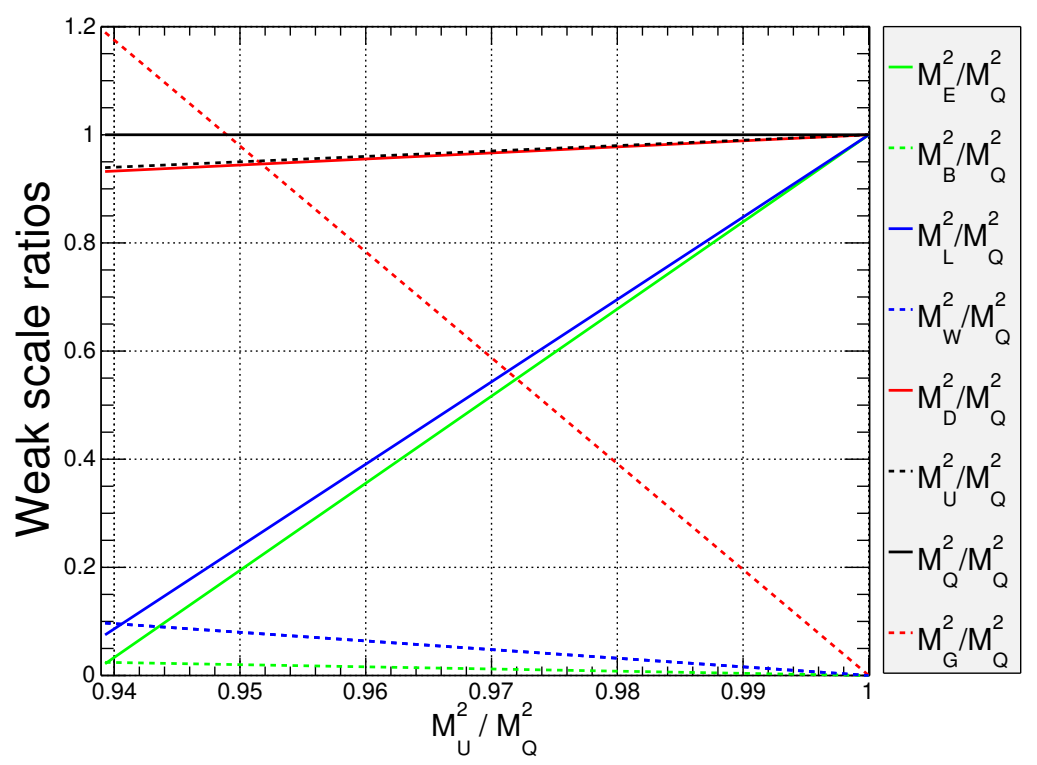

Figure 1. Dependence of the squared mass ratios $(4.9)-(4.14)$ on the parameter $\frac{M_{U}^{2}}{M_{Q}^{2}}$. We only show the physically viable range.

such restriction arises from the constraint $M_{E}^{2}>0$. Using eq. (4.11) this translates into

$$
\frac{M_{U}^{2}}{M_{Q}^{2}}>\frac{20 c_{1}-36 c_{3}}{35 c_{1}-36 c_{2}-36 c_{3}} \approx 0.94
$$

In addition, we should also require positivity of the gaugino mass squared boundary condition (4.8), which in turn implies

$$
\frac{M_{U}^{2}}{M_{Q}^{2}}<1
$$

Requiring that $M_{0}^{2}>0$ does not lead to any further restrictions, so that the physically allowed range for $\frac{M_{U}^{2}}{M_{Q}^{2}}$, depicted in figure 1 , is $(0.94,1)$.

For any given value of $\frac{M_{U}^{2}}{M_{Q}^{2}}$, the hierarchy of weak scale masses is uniquely determined. Furthermore, whenever a pair of lines in figure 1 cross, the hierarchy changes, since the mass ordering of the corresponding particles is reversed. Therefore, we can enumerate the hierarchies by examining the values of the independent degree of freedom $\frac{M_{U}^{2}}{M_{Q}^{2}}$ at which lines in figure 1 cross. Let

$$
\left.\frac{M_{U}^{2}}{M_{Q}^{2}}\right|_{M_{X}=M_{Y}}
$$

denote the value of $\frac{M_{U}^{2}}{M_{Q}^{2}}$ at which the $\frac{M_{X}^{2}}{M_{Q}^{2}}$ line and the $\frac{M_{Y}^{2}}{M_{Q}^{2}}$ line in figure 1 intersection It is 
easy to see that there are a total of eight intersection points, ordered as

$$
\begin{aligned}
& \left.\frac{M_{U}^{2}}{M_{Q}^{2}}\right|_{M_{B}=M_{E}}<\left.\frac{M_{U}^{2}}{M_{Q}^{2}}\right|_{M_{W}=M_{L}}<\left.\frac{M_{U}^{2}}{M_{Q}^{2}}\right|_{M_{W}=M_{E}}<\left.\frac{M_{U}^{2}}{M_{Q}^{2}}\right|_{M_{G}=M_{Q}}< \\
& <\left.\frac{M_{U}^{2}}{M_{Q}^{2}}\right|_{M_{G}=M_{U}}<\left.\frac{M_{U}^{2}}{M_{Q}^{2}}\right|_{M_{G}=M_{D}}<\left.\frac{M_{U}^{2}}{M_{Q}^{2}}\right|_{M_{G}=M_{L}}<\left.\frac{M_{U}^{2}}{M_{Q}^{2}}\right|_{M_{G}=M_{E}} .
\end{aligned}
$$

The eight points (4.18) divide the allowed $\frac{M_{U}^{2}}{M_{Q}^{2}}$ interval into 9 sub-intervals, where each sub-interval corresponds to one specific hierarchy. This confirms that the CMSSM indeed has 9 possible 8-particle hierarchies, as shown in table 3. Figure 1 reveals that they are (going from left to right):

$$
\begin{array}{lll}
G Q U D W L B E: & M_{G}>M_{Q}>M_{U}>M_{D}>M_{W}>M_{L}>M_{B}>M_{E}, \\
G Q U D W L E B: & M_{G}>M_{Q}>M_{U}>M_{D}>M_{W}>M_{L}>M_{E}>M_{B}, \\
G Q U D L W E B: & M_{G}>M_{Q}>M_{U}>M_{D}>M_{L}>M_{W}>M_{E}>M_{B}, \\
G Q U D L E W B: & M_{G}>M_{Q}>M_{U}>M_{D}>M_{L}>M_{E}>M_{W}>M_{B}, \\
\text { QGUDLEWB: } & M_{Q}>M_{G}>M_{U}>M_{D}>M_{L}>M_{E}>M_{W}>M_{B}, \\
\text { QUGDLEWB: } & M_{Q}>M_{U}>M_{G}>M_{D}>M_{L}>M_{E}>M_{W}>M_{B}, \\
\text { QUDGLEWB: } & M_{Q}>M_{U}>M_{D}>M_{G}>M_{L}>M_{E}>M_{W}>M_{B}, \\
\text { QUDLGEWB: } & M_{Q}>M_{U}>M_{D}>M_{L}>M_{G}>M_{E}>M_{W}>M_{B}, \\
\text { QUDLEGWB: } & M_{Q}>M_{U}>M_{D}>M_{L}>M_{E}>M_{G}>M_{W}>M_{B} .
\end{array}
$$

\section{Results: the set of forbidden $n$-particle (sub-)hierarchies}

Table 3 may seem an unedifying mélange of data. However, it turns out that if

1. one considers forbidden hierarchies rather than allowed hierarchies, and

2. inspects a forbidden $n$-particle hierarchy for forbidden $2,3, \ldots, n-1$-particle subhierarchies contained in it,

then the picture becomes much simpler. Our results in terms of forbidden hierarchies are shown in table 4 . The table is constructed as follows. For each model, we begin by considering the $8 \times 7=56$ possible 2-particle hierarchies. For each of these 2-particle sub-hierarchies, we check whether it is present in at least one of the allowed 8-particle hierarchies counted in the last column of table $3 .^{5}$ If the answer is "yes", then the 2particle sub-hierarchy is in principle allowed in this model, but if the answer is "no", this 2-particle sub-hierarchy will never appear in the model, and is therefore categorized as "forbidden". The total number of forbidden 2-particle sub-hierarchies is then tallied up and listed in the $n=2$ column of table 4 , while the explicit lists of the forbidden 2-particle

\footnotetext{
${ }^{5}$ Equivalently, we can directly check whether the 2-particle sub-hierarchy under consideration is one of the allowed 2-particle sub-hierarchies from the $n=2$ column of table 3 .
} 


\begin{tabular}{|c|r|r|r|r|r|r|r|}
\hline \multirow{2}{*}{$\begin{array}{c}\text { Case } \\
(m)\end{array}$} & \multicolumn{7}{|c|}{$n$} \\
\cline { 2 - 8 } & 2 & 3 & 4 & 5 & 6 & 7 & 8 \\
\hline 1 & 20 & 0 & 12 & 0 & 0 & 0 & 0 \\
\hline 2 & 14 & 0 & 13 & 0 & 2 & 0 & 0 \\
\hline 3 & 7 & 36 & 34 & 10 & 3 & 0 & 0 \\
\hline 4 & 5 & 18 & 79 & 14 & 3 & 0 & 0 \\
\hline 5 & 1 & 6 & 121 & 168 & 149 & 12 & 0 \\
\hline 6 & 0 & 6 & 45 & 216 & 288 & 98 & 3 \\
\hline 7 & 0 & 0 & 89 & 176 & 426 & 434 & 22 \\
\hline 8 & 0 & 0 & 32 & 148 & 809 & 398 & 54 \\
\hline
\end{tabular}

Table 4. The number of irreducible forbidden $n$-particle hierarchies for each of the eight models considered in table 2 .

hierarchies appear in appendix A, see eqs. (A.1), (A.3), (A.6), (A.11), and (A.13). Clearly, for any given model $m$, a specific 2-particle sub-hierarchy is either allowed or forbidden, so the total number $N_{2 \text {,forbidden }}^{(m)}$ of forbidden $n=2$ sub-hierarchies in the model and the total number $N_{2 \text {,allowed }}^{(m)}$ of allowed $n=2$ sub-hierarchies in the model add up to the total number of 2-particle sub-hierarchies:

$$
N_{2, \text { forbidden }}^{(m)}+N_{2, \text { allowed }}^{(m)}=56
$$

which is seen to hold for the results in the respective columns in tables 3 and 4 . We also observe that as the number $d^{(m)}$ of GUT-scale inputs increases, the number $N_{2 \text {,forbidden }}^{(m)}$ of forbidden 2-particle sub-hierarchies decreases. In particular, for models 6,7 , and 8 , we find that all 2-particle sub-hierarchies are possible. At the same time, in the most constrained model, the CMSSM, there are 20 forbidden 2-particle hierarchies, which will be discussed in detail in section 5.1.

Having thus determined the set of all forbidden 2-particle sub-hierarchies, we now move on to finding the set of all forbidden 3-particle sub-hierarchies. Clearly, a large number of 3-particle sub-hierarchies will be forbidden simply because they contain a forbidden 2particle sub-hierarchy. Such cases are uninteresting to us, and we focus only on the search for irreducible forbidden 3-particle hierarchies, i.e., 3-particle sub-hierarchies which are composed of allowed 2-particle sub-hierarchies only. The number $N_{3 \text {,forbidden }}^{(m)}$ of such "newly forbidden" 3-particle sub-hierarchies is listed in the third column of table 4. Interestingly, the results for $N_{3 \text {,forbidden }}^{(m)}$ do not follow quite the same pattern that we already observed

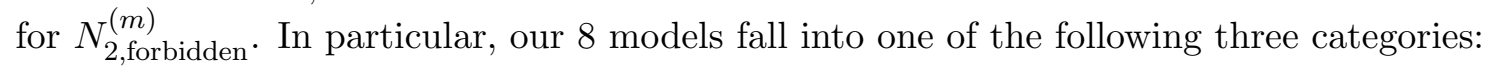

- Models in which in principle there exist forbidden 3-particle sub-hierarchies, but none of them is "newly forbidden". In other words, all forbidden 3-particle sub-hierarchies already contain a forbidden 2-particle sub-hierarchy. According to table 4, models $m=1$ and $m=2$ are of this type. 
- Models in which there are no forbidden 3-particle sub-hierarchies to begin with. These are the models with a relatively large number of input parameters, namely models $m=7$ and $m=8$.

- Lastly, there are models in which we obtain non-trivial results at the level of 3-particle hierarchies. In models $m=3, m=4, m=5$ and $m=6$, there arise newly forbidden 3 -particle sub-hierarchies, which cannot be explained simply with the presence of a forbidden 2-particle sub-hierarchy. The number of "newly forbidden" 3-particle subhierarchies does follow the expected pattern - generally speaking, there are more such hierarchies for the models with fewer input parameters.

Having accounted for the forbidden 2-particle sub-hierarchies and the newly forbidden 3-particle sub-hierarchies, we then proceed to investigate the number $N_{4, \text { forbidden }}^{(m)}$ of newly forbidden 4-particle sub-hierarchies. The results are listed in the fourth column of table 4. Unlike the case of 3-particle sub-hierarchies, we find that now all models predict newly forbidden (4-particle) sub-hierarchies. In the case of models $m=7$ and $m=8$, this is the first time we encounter forbidden sub-hierarchies.

The process just described continues until we fill out the whole table 4. Comparing our results in table 3 for the allowed sub-hierarchies and the complementary results in table 4 for the forbidden sub-hierarchies, we see significant simplification in the latter case in terms of counting hierarchies and bookkeeping. At the same time, it is worth emphasizing that tables 3 and 4 are simply two different ways to present our results on the restrictions imposed by various models on particle mass hierarchies. Whether we use the language of allowed or forbidden hierarchies, the amount of presented information remains the same.

However, in practice there may be situations where one language is preferred over the other. For example, consider an initial discovery in which only a few supersymmetric particles have been observed. Let us focus on the simplest and most likely scenario where only two particles are detected: a lighter particle $X$ with mass $M_{X}$, and a heavier partner $Y$ with mass $M_{Y}\left(M_{Y}>M_{X}\right)$. Given just this single piece of information, what can one conclude about the various models from table 2 ? The answer is given in table 5 , which lists all possible 2-particle hierarchies, and the corresponding models in which a particular hierarchy is forbidden. There are several conclusions which can be drawn from the table:

- Models $m=6, m=7$ and $m=8$ are completely absent from table 5 , which simply reflects the fact that in table 4 we did not find any forbidden 2-particle hierarchies for those models. This means that the discovery of just two supersymmetric particles (no matter what they are) is insufficient to rule out SUSY models with large number of parameters like models $m=6,7,8$.

- There are 36 2-particle sub-hierarchies which are present in all models - the 28 cases above the diagonal in table 5, plus 8 more cases below the diagonal. If the data happens to be one of those 2-particle hierarchies, we will not be able to make any definitive statements about the eight theory models since they will all still be allowed.

- The 2-particle hierarchies in which the heavier particle is colored while the lighter particle is not, are not restrictive at all. This is a well-known feature of SUGRA-type 


\begin{tabular}{|c|c|c|c|c|c|c|c|c|c|}
\hline \multirow{2}{*}{$Y X$} & \multicolumn{9}{|c|}{$X$} \\
\cline { 2 - 10 }$\left(M_{Y}>M_{X}\right)$ & $Q$ & $U$ & $D$ & $L$ & $E$ & $G$ & $W$ & $B$ \\
\hline \multirow{4}{*}{$Y$} & - & & & & & & & \\
\hline & $U$ & 1,2 & - & & & & & & \\
\hline & $D$ & 1 & 1,5 & - & & & & & \\
\cline { 2 - 11 } & $L$ & 1 & 1 & 1,2 & - & & & & \\
\cline { 2 - 11 } & $E$ & 1,2 & 1,2 & 1 & 1 & - & & & \\
\hline & & & & & & & - & & \\
\hline & $W$ & $1,2,3,4$ & 1,2 & $1,2,3$ & & & $1,2,3,4$ & - & \\
\hline & $W$ & $1,2,3,4$ & 1,2 & $1,2,3$ & 1,2 & & $1,2,3,4$ & $1,2,3,4$ & - \\
\hline
\end{tabular}

Table 5. The 56 possible 2-particle hierarchies $Y X$ (with $M_{Y}>M_{X}$ ), where the heavier (lighter) particle is shown in the corresponding row (column) of the table. Each box in the table (except for the boxes along the diagonal) represents one of the 56 possible 2-particle hierarchies, and the entries inside each box indicate the model numbers $m$ in which that hierarchy is forbidden.

scenarios, in which the RGE evolution tends to split the spectrum in such a way that the colored superpartners are heavier than their electroweak counterparts. In particular, in all eight models, the gluino can be the heavier of the two particles. Similarly, in all models, the right-handed selectron can be the lighter of the two particles.

- Among the most restrictive 2-particle hierarchies are those in which the lighter particle is colored, while the heavier particle is not. For example, observing $W Q, B Q$, $W G$ or $B G$ rules out four of the models right away. If both particles happen to be colored (or both are uncolored), then restrictive 2-particle hierarchies are those in which the lighter particle is charged under $\mathrm{SU}(2)$ while the heavier one is not, e.g., $B W, B L, U Q, D Q$.

The python code described in appendix B not only generates all allowed hierarchies described in the previous section, but it can also easily reproduce the results on forbidden hierarchies discussed in the current section. For details on how to use the code and its functionality we refer the reader to appendix B. We shall conclude this section by considering a few illustrative examples which allow analytical treatment by means of sum rules.

\subsection{Identifying all forbidden hierarchies in the CMSSM}

As seen in table 4, the CMSSM is characterized by 20 forbidden 2-particle sub-hierarchies and 12 newly forbidden 4-particle sub-hierarchies. These forbidden sub-hierarchies are explicitly listed in eqs. (A.1) and (A.2), respectively. Here we shall demonstrate how to re-derive those results with the help of the method of mass sum rules [61]. 
Our starting point is the relation between the weak-scale and GUT-scale parameters,

$$
\left(\begin{array}{c}
M_{Q}^{2} \\
M_{U}^{2} \\
M_{D}^{2} \\
M_{L}^{2} \\
M_{E}^{2} \\
M_{G}^{2} \\
M_{W}^{2} \\
M_{B}^{2}
\end{array}\right)=\left(\begin{array}{ll}
1 & 6.97 \\
1 & 6.54 \\
1 & 6.49 \\
1 & 0.52 \\
1 & 0.15 \\
0 & 8.29 \\
0 & 0.68 \\
0 & 0.17
\end{array}\right)\left(\begin{array}{c}
M_{0}^{2} \\
M_{1 / 2}^{2}
\end{array}\right) .
$$

The last three equations express the gaugino masses in terms of a single parameter, $M_{1 / 2}$. Noting the different proportionality coefficients, it is easy to see that the CMSSM predicts the gaugino mass hierarchy

$$
M_{G}^{2}>M_{W}^{2}>M_{B}^{2}
$$

This observation immediately rules out the three 2-particle hierarchies

$$
\{B W, W G, B G\} \text {. }
$$

Another way to arrive at this result is to note that one can eliminate the $M_{1 / 2}$ parameter and obtain two independent gaugino mass sum rules, e.g.

$$
\begin{aligned}
& 0.68 M_{G}^{2}-8.29 M_{W}^{2}=0, \\
& 0.17 M_{W}^{2}-0.68 M_{B}^{2}=0,
\end{aligned}
$$

which can be equivalently written as

$$
\begin{aligned}
-0.68\left(M_{W}^{2}-M_{G}^{2}\right)-7.61 M_{W}^{2} & =0, \\
-0.17\left(M_{B}^{2}-M_{W}^{2}\right)-0.51 M_{B}^{2} & =0 .
\end{aligned}
$$

Note that the numerical coefficients on the left-hand side of those equations are all negative. Thus the hierarchy $W G$, where $M_{W}>M_{G}$, violates the first sum rule, eq. (5.7), while the hierarchy $B W$, where $M_{B}>M_{W}$, is in contradiction with the second sum rule, eq. (5.8). ${ }^{6}$

Similar logic can be applied to the sfermion sector, where the dependence on the parameter $M_{0}^{2}$ is the same for all sfermion masses squared, and the mass splittings are induced only due to the different dependence on $M_{1 / 2}^{2}$. The CMSSM predicts the scalar masses in the order

$$
M_{Q}^{2}>M_{U}^{2}>M_{D}^{2}>M_{L}^{2}>M_{E}^{2},
$$

which rules out ten additional 2-particle hierarchies, namely

$$
\{E L, E D, E U, E Q, L D, L U, L Q, D U, D Q, U Q\} \text {. }
$$

\footnotetext{
${ }^{6}$ The impossibility of the third forbidden gaugino mass hierarchy, $B G$, follows from a linear combination of eqs. (5.5) and (5.6), e.g.$$
0.17 M_{G}^{2}-8.29 M_{B}^{2}=-0.17\left(M_{B}^{2}-M_{G}^{2}\right)-8.12 M_{B}^{2}=0 .
$$ 
This conclusion can also be justified with suitable sum rules, e.g. $E L$ is ruled out by the relation

$$
-0.17\left(M_{E}^{2}-M_{L}^{2}\right)-0.37 M_{B}^{2}=0 .
$$

Eqs. (5.4) and (5.10) account for 13 of the 20 forbidden 2-particle hierarchies. The remaining seven forbidden hierarchies, namely

$$
\{W Q, B Q, W U, B U, W D, B D, B L\}
$$

can also be understood in terms of sum rules involving two sfermion masses and one gaugino mass:

$$
\begin{aligned}
W Q: & -6.82\left(M_{W}^{2}-M_{Q}^{2}\right)-6.14 M_{Q}^{2}-0.68 M_{E}^{2}=0, \\
B Q: & -6.82\left(M_{B}^{2}-M_{Q}^{2}\right)-6.65 M_{Q}^{2}-0.17 M_{E}^{2}=0, \\
W U: & -6.39\left(M_{W}^{2}-M_{U}^{2}\right)-5.71 M_{U}^{2}-0.68 M_{E}^{2}=0, \\
B U: & -6.39\left(M_{B}^{2}-M_{U}^{2}\right)-6.22 M_{U}^{2}-0.17 M_{E}^{2}=0, \\
W D: & -6.34\left(M_{W}^{2}-M_{D}^{2}\right)-5.66 M_{D}^{2}-0.68 M_{E}^{2}=0, \\
B D: & -6.34\left(M_{B}^{2}-M_{D}^{2}\right)-6.17 M_{D}^{2}-0.17 M_{E}^{2}=0, \\
B L: & -0.37\left(M_{B}^{2}-M_{L}^{2}\right)-0.20 M_{L}^{2}-0.17 M_{E}^{2}=0 .
\end{aligned}
$$

As indicated in table 4, in the CMSSM model the forbidden 2-particle hierarchies, eqs. (5.4), (5.10) and (5.12), completely specify the forbidden 3-particle hierarchies as well. However, there still remain twelve newly forbidden 4-particle hierarchies which do not contain any forbidden 2-particle hierarchies and need to be motivated by a different argument.

The idea is the following. Recall from figure 1 that in the allowed range for $\frac{M_{U}^{2}}{M_{Q}^{2}}$ there exist eight crossing points (4.17) where we switch from a hierarchy $X Y$ (to the left of the crossing point) to a hierarchy $Y X$ (to the right of the crossing point). Now consider two such crossing points, $X_{1} Y_{1} \rightarrow Y_{1} X_{1}$ and $X_{2} Y_{2} \rightarrow Y_{2} X_{2}$, where all four particles $X_{1}, X_{2}, Y_{1}$ and $Y_{2}$ are different. For definiteness let us also assume that

$$
\left.\frac{M_{U}^{2}}{M_{Q}^{2}}\right|_{M_{X_{1}}=M_{Y_{1}}}<\left.\frac{M_{U}^{2}}{M_{Q}^{2}}\right|_{M_{X_{2}}=M_{Y_{2}}} .
$$

Now consider the 4-particle hierarchy $Y_{2} X_{2} X_{1} Y_{1}$. The $X_{1} Y_{1}$ bit requires

$$
\frac{M_{U}^{2}}{M_{Q}^{2}}<\left.\frac{M_{U}^{2}}{M_{Q}^{2}}\right|_{M_{X_{1}}=M_{Y_{1}}}
$$

while the $Y_{2} X_{2}$ bit in turn requires

$$
\left.\frac{M_{U}^{2}}{M_{Q}^{2}}\right|_{M_{X_{2}}=M_{Y_{2}}}<\frac{M_{U}^{2}}{M_{Q}^{2}} .
$$


In light of our original assumption (5.20), eqs. (5.21) and (5.22) place contradictory requirements on the parameter $\frac{M_{U}^{2}}{M_{Q}^{2}}$, thus the hierarchy $Y_{2} X_{2} X_{1} Y_{1}$ is not allowed.

From (4.18) we find a total of 13 pairs of crossing points for which all four particles involved are different. This gives us 13 possible candidates for newly forbidden 4-particle hierarchies:

$$
\begin{aligned}
& \{Q G B E, Q G W L, Q G W E, U G B E, U G W L, U G W E, \\
& D G B E, D G W L, D G W E, L G B E, L G W E, L W B E, E G L W\} .
\end{aligned}
$$

However, the last 4-particle hierarchy, $E G L W$, contains a forbidden 2-particle sub-hierarchy, $E L$, and is therefore not newly forbidden. This leaves us with exactly 12 newly forbidden 4-particle hierarchies, which are precisely those listed in (A.2).

\subsection{Examples of forbidden hierarchies in the most general case $(m=8)$}

In the previous subsection (5.1) we discussed the classification of forbidden hierarchies in a simple case like the CMSSM model. We shall now consider the other extreme, namely, the most general case of model $m=8$, where all 6 GUT scale input parameters are a priori unconstrained. According to table 4, in the case of model $m=8$, there are 32 forbidden 4-particle hierarchies, which are listed in eq. (A.18). In this subsection we shall illustrate a few specific examples.

As we saw in the case of the CMSSM, a mass hierarchy may be forbidden because it violates a mass sum rule. In the case of model $m=8$, there are 6 input model parameters, which enforces two mass sum rules among the 8 observable mass parameters, e.g.

$$
\begin{aligned}
M_{Q}^{2}+M_{D}^{2}-M_{L}^{2}-M_{E}^{2}-1.56 M_{G}^{2}+0.99 M_{B}^{2} & =0, \\
M_{U}^{2}+2 M_{D}^{2}-2 M_{L}^{2}-M_{E}^{2}-2.34 M_{G}^{2}+1.44 M_{W}^{2}+0.74 M_{B}^{2} & =0,
\end{aligned}
$$

\subsubsection{The mass hierarchy $G W Q B$}

Let us first consider the forbidden 4-particle hierarchy $G W Q B$ and rewrite the sum rules (5.24) and (5.25) in terms of the positive mass differences, e.g. $M_{G}^{2}-M_{W}^{2}, M_{W}^{2}-M_{Q}^{2}$ and $M_{Q}^{2}-M_{B}^{2}$ :

$$
\begin{array}{r}
-1.56\left(M_{G}^{2}-M_{Q}^{2}\right)-0.56\left(M_{Q}^{2}-M_{B}^{2}\right)+0.42 M_{B}^{2}+M_{D}^{2}-M_{L}^{2}-M_{E}^{2}=0 \\
-2.34\left(M_{G}^{2}-M_{W}^{2}\right)-0.91\left(M_{W}^{2}-M_{B}^{2}\right)-0.17 M_{B}^{2}+M_{U}^{2}+2 M_{D}^{2}-2 M_{L}^{2}-M_{E}^{2}=0 .
\end{array}
$$

If this manipulation renders one of the sum rules (or a linear combination of them) in a form where all terms have numerical coefficients of the same sign, the hierarchy will be clearly forbidden, due to the sum rule. Unfortunately, this is not the case here - the coefficients in both (5.26) and (5.27) have alternating signs, and the mass parameters can be suitably adjusted to make the left-hand sides of those equations vanish. Therefore, the sub-hierarchy $G W Q B$ is not forbidden by the sum rules alone, and we need to further investigate the GUT scale boundary conditions $\vec{G}^{8}$. For this purpose, we shall invert eq. (3.12) and solve for $\vec{G}^{8}$ in terms of the weak-scale mass parameters. Because of the two sum rules (5.24) 
and (5.25), only 6 of the weak-scale mass-squared parameters are linearly independent, and one possible choice would be to supplement the masses of the 4 particles entering the hierarchy $G W Q B$ under consideration, with the two slepton masses squared $M_{L}^{2}$ and $M_{E}^{2}$. Then the relevant $d^{(8)}=6$ equations from the system (3.12) are

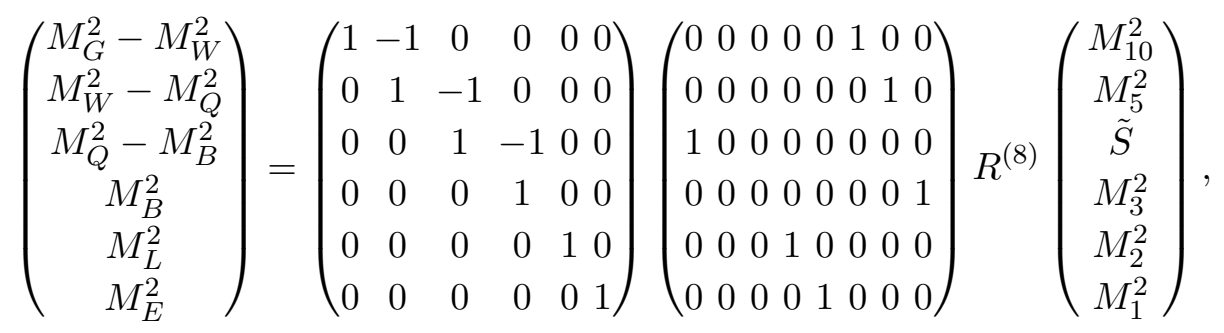

Inverting this equation, we find

$$
\left(\begin{array}{c}
M_{10}^{2} \\
M_{5}^{2} \\
\tilde{S} \\
M_{3}^{2} \\
M_{2}^{2} \\
M_{1}^{2}
\end{array}\right)=\left(\begin{array}{cccccc}
-0.94 & -1.8 & -0.6 & -0.45 & 0 & -0.2 \\
0.47 & 0.18 & -0.42 & -1.16 & 1 & 0.6 \\
0.16 & 0.3 & 0.1 & -0.07 & 0 & 0.2 \\
0.12 & 0.12 & 0.12 & 0.12 & 0 & 0 \\
0 & 1.48 & 1.48 & 1.48 & 0 & 0 \\
0 & 0 & 0 & 5.89 & 0 & 0
\end{array}\right)\left(\begin{array}{c}
M_{G}^{2}-M_{W}^{2} \\
M_{W}^{2}-M_{Q}^{2} \\
M_{Q}^{2}-M_{B}^{2} \\
M_{B}^{2} \\
M_{L}^{2} \\
M_{E}^{2}
\end{array}\right)
$$

where we have substituted numeric values. Note, in particular, the first equation, which specifies the value of the GUT scale parameter $M_{10}^{2}$ as

$$
M_{10}^{2}=-0.94\left(M_{G}^{2}-M_{W}^{2}\right)-1.8\left(M_{W}^{2}-M_{Q}^{2}\right)-0.6\left(M_{Q}^{2}-M_{B}^{2}\right)-0.45 M_{B}^{2}-0.2 M_{E}^{2} .
$$

Since all mass terms on the right-hand side have negative coefficients, the weak-scale masses squared are positive by definition, and the mass-squared differences are positive by the assumption of the hierarchy $G W Q B$, we conclude that the $G W Q B$ hierarchy necessarily requires a tachyonic value for $M_{10}^{2}$ and is therefore forbidden.

Recall that above we had the freedom of choosing two additional weak-scale parameters to form the vector in the left-hand side of (5.28). It is instructive to see what happens if we had chosen a different set of weak-scale mass parameters, say $M_{U}^{2}$ and $M_{L}^{2}$ instead. In that case eq. (5.28) is replaced with

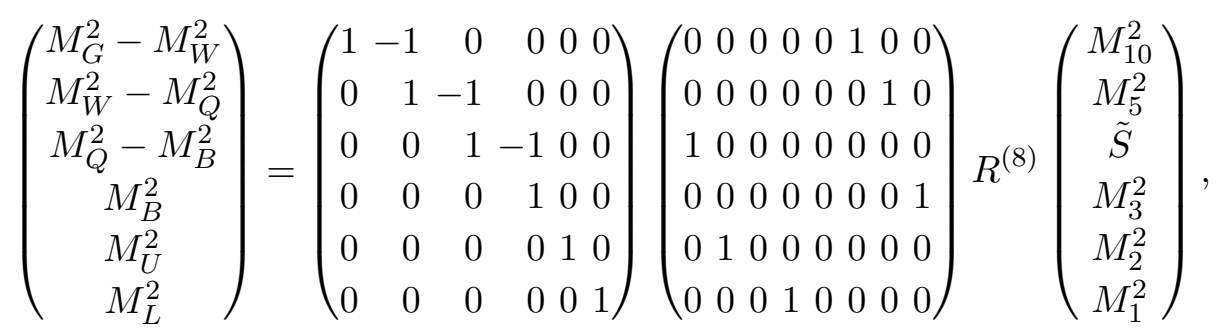


and inverting, we get

$$
\left(\begin{array}{c}
M_{10}^{2} \\
M_{5}^{2} \\
\tilde{S} \\
M_{3}^{2} \\
M_{2}^{2} \\
M_{1}^{2}
\end{array}\right)=\left(\begin{array}{rrrrrr}
-0.78 & -1.36 & -0.56 & -0.66 & 0.2 & 0 \\
0 & -1.15 & -0.55 & -0.55 & -0.6 & 1 \\
0 & -0.14 & 0.06 & 0.13 & -0.2 & 0 \\
0.12 & 0.12 & 0.12 & 0.12 & 0 & 0 \\
0 & 1.48 & 1.48 & 1.48 & 0 & 0 \\
0 & 0 & 0 & 5.89 & 0 & 0
\end{array}\right)\left(\begin{array}{c}
M_{G}^{2}-M_{W}^{2} \\
M_{W}^{2}-M_{Q}^{2} \\
M_{Q}^{2}-M_{B}^{2} \\
M_{B}^{2} \\
M_{U}^{2} \\
M_{L}^{2}
\end{array}\right) .
$$

Note that the matrix on the right hand side does not have all negative entries in any given row, so the contradiction is not immediately obvious. However, recall the existence of the two sum rules (5.26) and (5.27), which define the remaining two parameters $M_{D}^{2}$ and $M_{E}^{2}$ :

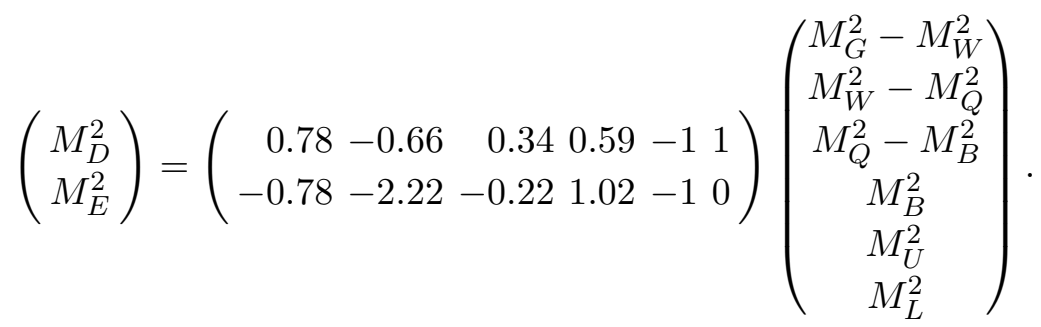

Putting together (5.32) and (5.33), we obtain

$$
\left(\begin{array}{c}
M_{10}^{2} \\
M_{5}^{2} \\
\tilde{S} \\
M_{3}^{2} \\
M_{2}^{2} \\
M_{1}^{2} \\
M_{D}^{2} \\
M_{E}^{2}
\end{array}\right)=\left(\begin{array}{rrrrrr}
-0.78 & -1.36 & -0.56 & -0.66 & 0.2 & 0 \\
0 & -1.15 & -0.55 & -0.55 & -0.6 & 1 \\
0 & -0.14 & 0.06 & 0.13 & -0.2 & 0 \\
0.12 & 0.12 & 0.12 & 0.12 & 0 & 0 \\
0 & 1.48 & 1.48 & 1.48 & 0 & 0 \\
0 & 0 & 0 & 5.89 & 0 & 0 \\
0.78 & -0.66 & 0.34 & 0.59 & -1 & 1 \\
-0.78 & -2.22 & -0.22 & 1.02 & -1 & 0
\end{array}\right)\left(\begin{array}{c}
M_{G}^{2}-M_{W}^{2} \\
M_{W}^{2}-M_{Q}^{2} \\
M_{Q}^{2}-M_{B}^{2} \\
M_{B}^{2} \\
M_{U}^{2} \\
M_{L}^{2}
\end{array}\right) .
$$

From a linear combination of the first and last equation, we obtain:

$$
\begin{aligned}
2 M_{10}^{2}+M_{E}^{2}= & -2.34\left(M_{G}^{2}-M_{W}^{2}\right)-4.93\left(M_{W}^{2}-M_{Q}^{2}\right) \\
& -1.33\left(M_{Q}^{2}-M_{B}^{2}\right)-0.3 M_{B}^{2}-0.6 M_{U}^{2} .
\end{aligned}
$$

This sum rule cannot be satisfied because the l.h.s. must be positive while the r.h.s. is clearly negative. The lesson is that, depending on our choice of parameters for the inversion, we may have to use the sum rules (5.26) and (5.27) in order to show that a hierarchy is forbidden.

\subsubsection{The mass hierarchy WQGL}

As another example, consider the 4-particle sub-hierarchy WQGL. The RGE solutions are

$$
\left(\begin{array}{c}
M_{W}^{2}-M_{Q}^{2} \\
M_{Q}^{2}-M_{G}^{2} \\
M_{G}^{2}-M_{L}^{2} \\
M_{L}^{2} \\
M_{U}^{2} \\
M_{E}^{2}
\end{array}\right)=\left(\begin{array}{rrrrrr}
-1 & 0 & -1 & -6.48 & 0.19 & -0.004 \\
1 & 0 & 1 & -1.81 & 0.49 & 0.004 \\
0 & -1 & 3 & 8.29 & -0.49 & -0.04 \\
0 & 1 & -3 & 0 & 0.49 & 0.04 \\
1 & 0 & -4 & 6.48 & 0 & 0.07 \\
1 & 0 & 6 & 0 & 0 & 0.15
\end{array}\right)\left(\begin{array}{c}
M_{10}^{2} \\
M_{5}^{2} \\
\tilde{S} \\
M_{3}^{2} \\
M_{2}^{2} \\
M_{1}^{2}
\end{array}\right) .
$$


Selecting $M_{U}^{2}$ and $M_{E}^{2}$ as additional parameters to invert the RGE equation, we have

$$
\left(\begin{array}{c}
M_{10}^{2} \\
M_{5}^{2} \\
\tilde{S} \\
M_{3}^{2} \\
M_{2}^{2} \\
M_{1}^{2}
\end{array}\right)=\left(\begin{array}{rrrrrr}
-0.69 & 0.27 & -0.57 & -0.57 & 0.12 & -0.08 \\
-1.15 & -0.55 & -0.55 & 0.45 & -0.6 & 0 \\
-0.06 & 0.02 & 0.07 & 0.07 & -0.14 & 0.06 \\
0 & 0 & 0.12 & 0.12 & 0 & 0 \\
1.48 & 1.48 & 1.48 & 1.48 & 0 & 0 \\
6.86 & -2.68 & 1.05 & 1.05 & 4.77 & 4.77
\end{array}\right)\left(\begin{array}{c}
M_{W}^{2}-M_{Q}^{2} \\
M_{Q}^{2}-M_{G}^{2} \\
M_{G}^{2}-M_{L}^{2} \\
M_{L}^{2} \\
M_{U}^{2} \\
M_{E}^{2}
\end{array}\right) .
$$

Again, we do not have a single row with negative coefficients, but forming the sum of $M_{10}^{2}$ and $M_{5}^{2}$ we find

$$
\begin{aligned}
M_{10}^{2}+M_{5}^{2}=- & 1.84\left(M_{W}^{2}-M_{Q}^{2}\right)-0.28\left(M_{Q}^{2}-M_{G}^{2}\right) \\
& -1.12\left(M_{G}^{2}-M_{L}^{2}\right)-0.12 M_{L}^{2}-0.48 M_{U}^{2}-0.08 M_{E}^{2} .
\end{aligned}
$$

This sum rule cannot be satisfied, since all terms in the r.h.s. have negative coefficients. Therefore, the hierarchy $W Q G L$ is forbidden.

\section{Conclusions and summary}

The search for SUSY is the paramount experimental challenge for Run II at the LHC. Once SUSY is discovered, it may provide invaluable clues about GUT-scale physics. The measured pattern of SUSY particle masses will play an important role in this quest, as we have explained above. Our proposal is to consider the relative ordering, or the "hierarchy", of the measured sparticle masses. By analyzing the one loop SUSY RGE's, it is relatively straightforward to derive the complete set of allowed hierarchies for a given choice of GUT scale boundary conditions. In this paper we considered hierarchies involving up to eight weak scale masses (first/second generation masses for each of the five sfermion families, and three gaugino masses), and analyzed eight different GUT-scale model scenarios (table 2). Our results are listed in appendix A and can be reproduced with the accompanying Python code described in appendix B. We also provided some intuitive arguments, based on mass sum rules and linear algebra tricks, to better understand and justify those results.

The advantage of the approach presented in this paper is that it allows to draw definitive conclusions based on only partial information. In particular, we have seen that the knowledge of a sub-hierarchy of a few sparticle masses, measured in the very early days of discovery, is sometimes sufficient to rule out a specific model. Consider, for example, the most general model, $m=8$, which has 6 GUT-scale input parameters. In principle, one would need 6 independent measurements in order to fully reconstruct the GUT-scale physics. On the other hand, we have shown that in this model, there are as many as 32 forbidden 4-particle sub-hierarchies. Therefore, the $m=8$ model can be ruled out with only 4 suitable measurements, if the data happens to point to one of the 32 forbidden hierarchies. This procedure is pictorially illustrated in figure 2 .

The analysis presented here can be extended in many directions. Obviously, one may consider more of the MSSM particles, or generalize to non-minimal models like the NMSSM. 


\section{SUSY Models}

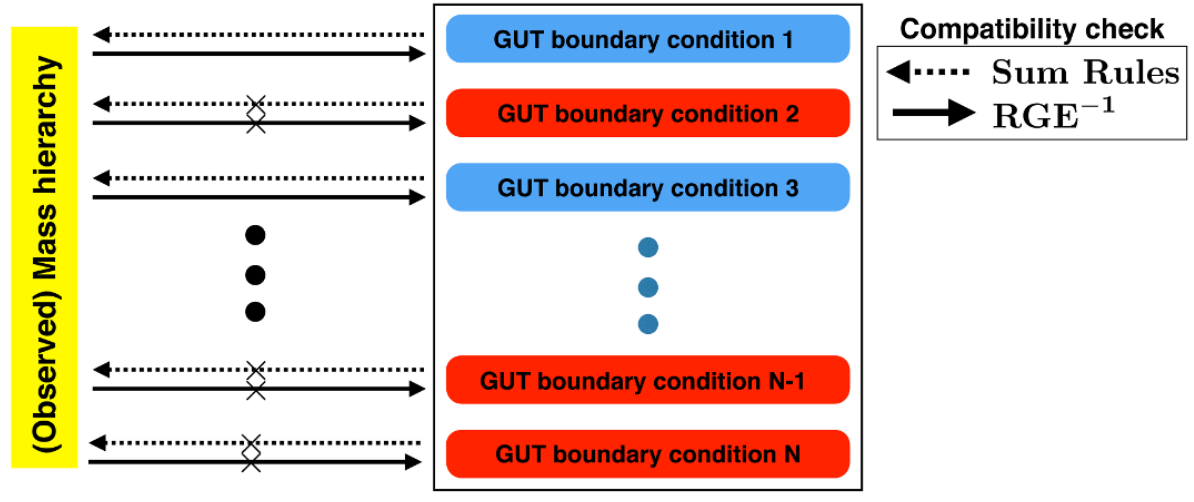

Figure 2. A schematic view on how hierarchies shed light on GUT-scale boundary conditions. Once a set of SUSY particle masses is observed (left), GUT-scale boundary conditions (center) incapable of producing this sparticle mass hierarchy are ruled out by querying our database. This conclusion can be independently verified by suitable mass sum rules or explicit running of the RGEs (right).

Our approach is not limited to SUGRA-type boundary conditions and can be applied in other SUSY breaking scenarios as well. Armed with the toolkit of allowed and forbidden hierarchies, we look forward to analyzing GUT scale models after a SUSY discovery at the LHC!

\section{Acknowledgments}

We thank P. Konar and G. Sarangi for collaboration in the initial stages of this work and A. de Gouvea and I. Low for useful comments and suggestions. MP thanks the high energy theory group at the University of Florida for its warm and humid hospitality. MP is supported by the Korea Ministry of Science, ICT and Future Planning, GyeongsangbukDo and Pohang City for Independent Junior Research Groups at the Asia Pacific Center for Theoretical Physics. MP is also supported by World Premier International Research Center Initiative (WPI Initiative), MEXT, Japan. Work also supported by DOE Grant No. DE-SC0010296.

\section{A Results on forbidden hierarchies}

In this appendix, we provide explicit lists of "irreducibly-forbidden" or "newly-forbidden" $n$-particle sub-hierarchies in various GUT scale scenarios ("models"). ${ }^{7}$ These are the forbidden $n$-particle hierarchies which do not contain a forbidden sub-hierarchy.

\section{A.1 Model $m=1$ (CMSSM)}

The forbidden (and hence irreducibly-forbidden) 2-particle hierarchies are

$$
\begin{aligned}
& W U, B W, D Q, B L, E D, B U, W Q, L Q, E Q, E L, \\
& L D, D U, W D, B G, W G, B Q, L U, E U, B D, U Q .
\end{aligned}
$$

\footnotetext{
${ }^{7}$ For the definition of the eight models, see table 2.
} 
The irreducibly-forbidden 4-particle hierarchies are

$$
\begin{aligned}
& U G B E, Q G W E, U G W L, L W B E, Q G W L, D G B E, \\
& D G W L, Q G B E, L G W E, L G B E, U G W E, D G W E .
\end{aligned}
$$

The are no newly forbidden $n$-particle hierarchies for $n \geq 5$ in this model. It is astonishing that all the information about the 8 ! possible hierarchies in the CMSSM is encoded in the two lists (A.1) and (A.1).

\section{A.2 Model $m=2$}

For model 2, the forbidden 2-particle hierarchies are

$$
W U, B W, B L, B U, W Q, E Q, L D, W D, B G, W G, B Q, E U, B D, U Q \text {. }
$$

The newly forbidden 4-particle hierarchies are

$$
\begin{aligned}
& G D L U, U G B E, D Q W L, Q G W E, G D L Q, G U E D, D U E L, \\
& D G W L, Q G B E, U G W E, Q D L U, D Q E L, G Q E D ;
\end{aligned}
$$

and the newly forbidden 6-particle hierarchies are

$$
U D L W B E, Q G D U W L \text {. }
$$

There are no other newly forbidden hierarchies.

\section{A.3 Model $m=3$ (NUHM)}

For this model, the forbidden 2-particle hierarchies are

$$
B W, W Q, W D, B G, W G, B Q, B D ;
$$

the newly-forbidden 3-particle hierarchies are

$$
\begin{aligned}
& D U Q, U E Q, E Q D, L U Q, U D Q, L E U, G D Q, E U D, E L Q, L U D, E L D, L Q U, \\
& L E Q, E U Q, W E U, D G Q, L E D, L U E, B E U, L G Q, D L Q, W U E, E G Q, L G D \\
& E G D, Q E D, L D Q, B U E, G L D, G E D, U E D, L D U, B L E, G L Q, B E L, G E Q
\end{aligned}
$$

the newly forbidden 4-particle hierarchies are

$$
\begin{aligned}
& D Q B E, G D L U, U G B L, D E B U, U Q W L, D U B E, Q D E U, D E L U, G Q L U, \\
& G D W U, G Q B U, G D E U, U G W L, D E W U, Q E W U, W E B L, G E B U, Q E L U, \\
& G Q E U, G D B U, G Q W U, G E L U, Q E B U, U D B L, Q D W U, U D E L, U Q E L, \\
& Q D L U, G E W U, Q U L D, D Q W E, Q D B U, U Q B L, D Q L E ;
\end{aligned}
$$

the newly forbidden 5-particle hierarchies are

$$
\begin{aligned}
& Q G W E L, Q G U W E, D G W E L, Q G U B E, Q G W L E, \\
& D G U W E, Q U G W E, D U G W E, D G W L E, Q U G B E ;
\end{aligned}
$$

and the newly forbidden 6-particle hierarchies are

$$
Q G U D W L, Q D E G W L, Q D E G B L .
$$

There are no newly forbidden 7-particle or 8-particle hierarchies in this model. 


\section{A.4 Model $m=4$}

For this model, the forbidden 2-particle hierarchies are

$$
B W, W Q, B G, W G, B Q ;
$$

and the newly-forbidden 3-particle hierarchies are

$$
\begin{aligned}
& U E Q, W D U, B L D, B U D, E U Q, W E U, B E U, W U E, E G Q, \\
& B U E, W L D, B D U, B L E, W U D, B E L, B D L, W D L, G E Q .
\end{aligned}
$$

Additionally, as shown in table 4, there are 79 newly forbidden 4-particle hierarchies, 14 newly forbidden 5-particle hierarchies, and 3 newly forbidden 6-particle hierarchies. (There are no newly forbidden 7-particle or 8-particle hierarchies.) In order to save space, we do not list these hierarchies explicitly here, however they can be easily obtained from the python code described in appendix B.

\section{A.5 Model $m=5$}

There is only one forbidden 2-particle hierarchy

$$
D U,
$$

and six newly-forbidden 3-particle hierarchies:

$$
B G U, G W Q, W G Q, E G U, G B U, G E U \text {. }
$$

Additionally, as shown in table 4, there are 121 newly forbidden 4-particle hierarchies, 168 newly forbidden 5-particle hierarchies, 149 newly forbidden 6-particle hierarchies, and 12 newly forbidden 7-particle hierarchies. (There are no newly forbidden 8-particle hierarchies.) In order to save space, we do not list these hierarchies explicitly here, however they can be easily obtained from the python code described in appendix B.

\section{A.6 Model $m=6$}

In this model, all 2-particle hierarchies are allowed. The forbidden 3-particle hierarchies are:

$$
B G U, G W Q, E G U, G B U, G E U, W G Q \text {. }
$$

The newly-forbidden 4-particle hierarchies are

$$
\begin{aligned}
& U E W Q, W E U Q, W D L Q, L Q G D, W B U Q, L G Q D, U Q W E, W Q D L, W U L Q, \\
& U Q W L, D Q W L, U W Q E, G D L Q, W Q U L, U W E Q, G U E D, W U Q L, U B W Q, \\
& W D Q L, U W Q B, G U B Q, L G D Q, G Q W U, W U E Q, G Q W D, B U G E, U Q W B, \\
& W U Q B, D L W Q, D W Q L, D W L Q, G L Q D, E U G B, W U Q E, U W B Q, U W L Q, \\
& W U B Q, W L D Q, U W Q L, G U E Q, G U B D, W Q G L, G Q L D, G L D Q, W L U Q .
\end{aligned}
$$

Additionally, as shown in table 4, there are 216 newly forbidden 5-particle hierarchies, 288 newly forbidden 6-particle hierarchies, 98 newly forbidden 7-particle hierarchies, and 3 newly forbidden 8-particle hierarchies. In order to save space, we do not list these hierarchies explicitly here, however they can be easily obtained from the python code described in appendix B. 


\section{A.7 Model $m=7$}

In model 7, all 2-particle and 3-particle hierarchies are allowed. The forbidden 4-particle hierarchies are:

LQGU,WDQU, BGUE, GWQB, GULQ,WDEQ, DLQU, DWQB, DULQ,WDLQ, $L U G Q, D B E U, W G Q U, D E B U, G L W Q, W U L Q, L G U Q, U Q W L, W D B Q, D W U Q$, $W G L Q, D U B E, D B W Q, W Q U L, G Q L U, B E D U, W U G Q, L D E Q, G B U E, B G E U$, $B D U E, W G Q B, G B W Q, W U Q L, B E G U, W D Q L, G E B U, G B E U, W D Q B, G W B Q$, GQWU,LUDQ,DEWQ, GUWQ, DLEQ, DELQ,DWQE, DWEQ, GWUQ, BUGE, GWQL,WEDQ,WUDQ,DLWQ,WGQL, LEDQ, DWQL, DUWQ,DWLQ,WDQE, $D B U E, L G Q U, W D U Q, D L Q E, W B G Q, W L G Q, B D E U, L D U Q, B U D E, L Q D U$, $D Q W E, U W L Q, W G U Q, L D Q U, L D Q E, W L D Q, D L U Q, W B D Q, D W Q U, W G B Q$, $U W Q L, G W Q U, D Q L E, G W L Q, W Q G L, G L U Q, D W B Q, G L Q U, W L U Q$.

Additionally, as shown in table 4, there are 176 newly forbidden 5-particle hierarchies, 426 newly forbidden 6-particle hierarchies, 434 newly forbidden 7-particle hierarchies, and 22 newly forbidden 8-particle hierarchies. In order to save space, we do not list these hierarchies explicitly here, however they can be easily obtained from the python code described in appendix B.

\section{A.8 Model $m=8$}

In this model, all 2-particle and 3-particle hierarchies are allowed. The forbidden 4-particle hierarchies are:

$$
\begin{aligned}
& B G E U, G B W Q, W U Q L, G E B U, G W Q L, W B G Q, W G B Q, W G L Q, \\
& B E G U, W G Q L, G B E U, U W Q L, B G U E, G W Q B, W U G Q, G U W Q, \\
& U W L Q, W G Q B, W G U Q, W L U Q, U Q W L, W G Q U, G W B Q, G W U Q, \\
& G B U E, G W Q U, G W L Q, W U L Q, G L W Q, W Q G L, W L G Q, W Q U L .
\end{aligned}
$$

Additionally, as shown in table 4, there are 148 newly forbidden 5-particle hierarchies, 809 newly forbidden 6-particle hierarchies, 398 newly forbidden 7-particle hierarchies, and 54 newly forbidden 8-particle hierarchies. In order to save space, we do not list these hierarchies explicitly here, however they can be easily obtained from the python code described in appendix B.

\section{B Python package for studying hierarchies}

We have provided a Python [84] package in the supplemental material to the arXiv version of this paper. It consists of two required files, susy_hierarchy_methods.py, and allowed_hierarchies.py and two optional files, forbidden_hierarchies.py and newly_forbidden_hierarchies.py. These files should work with all versions of Python including and subsequent to Python 2.6. 
Information about which hierarchies are allowed or forbidden in various SUSY scenarios is contained in the files allowed_hierarchies.py and forbidden_hierarchies.py. Of course a hierarchy in a given SUSY scenario is either allowed or forbidden, so it is sufficient to have only, e.g., an enumeration of allowed hierarchies in various models. However, the generation of forbidden hierarchy information from allowed hierarchy information is relatively slow $\sim 1$ minute on a modern laptop, so we do include the, in principle redundant, files forbidden_hierarchies.py and newly_forbidden_hierarchies.py.

\section{B.1 Data structures in Python}

To explain how hierarchy data is stored in the files allowed_hierarchies.py,

forbidden_hierarchies.py, and newly_forbidden_hierarchies.py, we must briefly review a few of the data structures present in Python. A list consists of elements, in a fixed order, between the square brackets '[' and '['. Elements are indexed by integers, e.g., the first element of my_list is my_list [0]. A dictionary is like a list, but the elements are stored between curly brackets ' $\{$ ' and ' $\}$ ' and are indexed by arbitrary items called "keys". An element of a dictionary is accessed by its key. For example if

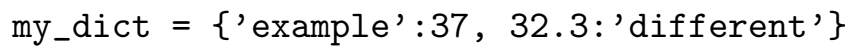

then

my_dict ['example'] $=37$

my_dict $\left[{ }^{\prime} 32.3^{\prime}\right]=$ 'different'

Finally a tuple is like a list, except that tuples cannot be modified (and tuples are enclosed in parentheses).

\section{B.2 Hierarchy data}

The file allowed_hierarchies.py contains the single command

allowed $=\left\{2:\left\{\left({ }^{\prime} L^{\prime},{ }^{\prime} W^{\prime}\right):[1,3,5,2,7,4,6,8],\left(Q^{\prime}, G^{\prime}\right): \backslash\right.\right.$

$[1,3,5,2,7,4,6,8]$, \ldots.

The dictionary "allowed" has keys $2,3, \ldots, 8$; these keys represent the number of particles in a hierarchy. The elements corresponding to each of these keys are dictionaries. The keys for these dictionary are the hierarchies, which are tuples of capital letters representing the particles in the hierarchy. The entry corresponding to a hierarchy is a list (in no particular order) of the SUSY scenarios in which the hierarchy is allowed. The numbering of SUSY scenarios or "cases" is that which is used throughout this paper and defined in table 2 . Note that only hierarchies which are allowed in some hierarchy have entries in "allowed". Thus

$\operatorname{print}($ allowed [2] [('U', 'Q')])

yields

$[3,5,7,4,6,8]$ 
as the 2-particle hierarchy ('U', 'Q') is allowed in models $3,4,5,6,7$, and 8 , while

print(allowed [4][('B', 'G', 'E', 'U')])

yields the error (in interactive session):

Traceback (most recent call last):

File "<stdin>", line 1, in <module>

KeyError: ('B', 'G', 'E', 'U')

as the 4-particle hierarchy ('B', 'G', 'E', 'U') is not allowed in any model.

The file forbidden_hierarchies.py uses the exact same format, but the dictionary is called "forbidden" and contains lists of the models in which hierarchies are forbidden, rather than allowed. Likewise the file newly_forbidden_hierarchies.py uses a dictionary called "'newly_forbidden' ', which tells us which hierarchies are newly forbidden (irreducible).

\section{B.3 Methods}

The Python expert can use the data in allowed_hierarchies.py, forbidden_hierarchies.py, and newly_forbidden_hierarchies.py for their own purposes. However, for those less familiar with Python or for those who would benefit from an example of methods (functions) which use the hierarchy data, we have provided another module (python file) with methods that process the data in allowed_hierarchies.py, forbidden_hierarchies.py, and newly_forbidden_hierarchies.py. These methods are

1. convert_to_standard_form( $\mathrm{s}$ )

This method translates a string, $s$, to a tuple of capital letters representing the SUSY particles in a hierarchy. This is the format of the keys representing hierarchies in our data arrays.

2. print_case (case)

This method prints a string describing the SUSY scenario ("case") labelled by the integer case, which must be between one and eight (inclusive).

3. get_info_on_hierarchy (s)

This method prints information listing and describing the SUSY scenarios ("cases") in which the hierarchy $s$ ( $s$ is a string, enclosed by single or double quotes) is allowed and in which it is forbidden.

4. number_of_N_hierarchies_in_case (data_type, $N$, case)

This method returns the number of $N$ particle hierarchies which are either allowed or forbidden (depending on whether the value of data_type is "allowed" or "forbidden") in SUSY scenario case.

5. list_of_N_hierarchies_in_case (data_type, $N$, case)

This method returns a list of $N$ particle hierarchies which are either allowed or forbidden (depending on whether the value of data_type is "allowed" or "forbidden") in SUSY scenario case. 
6. print_table_of_cases()

This method prints a table that describes the eight SUSY scenarios ("cases") that we consider.

7. print_table_of_number_of_hierarchies (data_type)

This method prints a table of all particle hierarchies which are either allowed or forbidden (depending on whether the value of data_type is "allowed" or "forbidden") in all eight SUSY scenarios.

\section{B.4 Using the code}

The Python expert can use the methods and as part of their own code by importing whichever modules they need. However, the simplest way to use the methods provided is to use Python in interactive mode. To do this, one types

python

in a terminal. While it is discouraged in general (as it negates useful features of Python in regard to namespaces), the simplest next step is to type

from susy_hierarchy_methods import *

into the Python interpreter. Note that the files susy_hierarchy_methods.py and allowed hierarchies.py must be in the current working directory, or must be in one's PYTHONPATH. Doing this also imports "allowed" from allowed_hierarchies.py automatically. If forbidden_hierarchies.py is in the working directory or the PYTHONPATH, then "forbidden" will be imported from this file. If not, "forbidden" will be generated automatically, though this can be somewhat time-consuming ( $\sim 1$ minute on a modern laptop). The situation regarding newly_forbidden_hierarchies.py is exactly analogous. One can then simply type the method one wishes to execute together with the appropriate arguments. Finally, we note that typing

help (name_of_method)

will provide a brief description of the method in question.

Open Access. This article is distributed under the terms of the Creative Commons Attribution License (CC-BY 4.0), which permits any use, distribution and reproduction in any medium, provided the original author(s) and source are credited.

\section{References}

[1] S.P. Martin, A supersymmetry primer, in Perspectives on supersymmetry II, G.L. Kane ed., pp. 1-153, Adv. Ser. Direct. High Energy Phys. 21 (2010) 1 [hep-ph/9709356] [InSPIRE].

[2] ATLAS collaboration, Observation of a new particle in the search for the Standard Model Higgs boson with the ATLAS detector at the LHC, Phys. Lett. B 716 (2012) 1 [arXiv: 1207.7214] [INSPIRE]. 
[3] CMS collaboration, Observation of a new boson at a mass of $125 \mathrm{GeV}$ with the CMS experiment at the LHC, Phys. Lett. B 716 (2012) 30 [arXiv:1207.7235] [INSPIRE].

[4] G. Jungman, M. Kamionkowski and K. Griest, Supersymmetric dark matter, Phys. Rept. 267 (1996) 195 [hep-ph/9506380] [INSPIRE].

[5] J.L. Feng, Naturalness and the Status of Supersymmetry, Ann. Rev. Nucl. Part. Sci. 63 (2013) 351 [arXiv:1302.6587] [INSPIRE].

[6] ATLAS Supersymmetry (SUSY) searches, https://twiki.cern.ch/twiki/bin/view/AtlasPublic/SupersymmetryPublicResults.

[7] CMS Supersymmetry Physics Results, https://twiki.cern.ch/twiki/bin/view/CMSPublic/PhysicsResultsSUS.

[8] T. Cohen and J.G. Wacker, Here be Dragons: The Unexplored Continents of the CMSSM, JHEP 09 (2013) 061 [arXiv: 1305.2914] [INSPIRE].

[9] I. Hinchliffe, F.E. Paige, M.D. Shapiro, J. Soderqvist and W. Yao, Precision SUSY measurements at CERN LHC, Phys. Rev. D 55 (1997) 5520 [hep-ph/9610544] [INSPIRE].

[10] CMS collaboration, Discovery potential for supersymmetry in CMS, J. Phys. G 28 (2002) 469 [hep-ph/9806366] [INSPIRE].

[11] M. Battaglia et al., Proposed post-LEP benchmarks for supersymmetry, Eur. Phys. J. C 22 (2001) 535 [hep-ph/0106204] [INSPIRE].

[12] B.C. Allanach et al., The Snowmass Points and Slopes: Benchmarks for SUSY searches, Eur. Phys. J. C 25 (2002) 113 [hep-ph/0202233] [INSPIRE].

[13] A. De Roeck, J.R. Ellis, F. Gianotti, F. Moortgat, K.A. Olive and L. Pape, Supersymmetric benchmarks with non-universal scalar masses or gravitino dark matter, Eur. Phys. J. C 49 (2007) 1041 [hep-ph/0508198] [INSPIRE].

[14] S.S. AbdusSalam et al., Benchmark Models, Planes, Lines and Points for Future SUSY Searches at the LHC, Eur. Phys. J. C 71 (2011) 1835 [arXiv:1109.3859] [InSPIRE].

[15] J.R. Ellis, J.L. Feng, A. Ferstl, K.T. Matchev and K.A. Olive, Prospects for detecting supersymmetric dark matter at post LEP benchmark points, Eur. Phys. J. C 24 (2002) 311 [astro-ph/0110225] [INSPIRE].

[16] G. Eigen, R. Gaitskell, G.D. Kribs and K.T. Matchev, Indirect investigations of supersymmetry, eConf C 010630 (2001) P342 [hep-ph/0112312] [INSPIRE].

[17] S. Arrenberg et al., Working Group Report: Dark Matter Complementarity, arXiv:1310.8621 [INSPIRE].

[18] C.F. Berger, J.S. Gainer, J.L. Hewett and T.G. Rizzo, Supersymmetry Without Prejudice, JHEP 02 (2009) 023 [arXiv:0812.0980] [INSPIRE].

[19] R.C. Cotta, J.S. Gainer, J.L. Hewett and T.G. Rizzo, Dark Matter in the MSSM, New J. Phys. 11 (2009) 105026 [arXiv:0903.4409] [INSPIRE].

[20] S.S. AbdusSalam, B.C. Allanach, F. Quevedo, F. Feroz and M. Hobson, Fitting the Phenomenological MSSM, Phys. Rev. D 81 (2010) 095012 [arXiv:0904.2548] [INSPIRE].

[21] S. Sekmen et al., Interpreting LHC SUSY searches in the phenomenological MSSM, JHEP 02 (2012) 075 [arXiv: 1109.5119] [INSPIRE]. 
[22] A. Arbey, M. Battaglia and F. Mahmoudi, Implications of LHC Searches on SUSY Particle Spectra: The pMSSM Parameter Space with Neutralino Dark Matter, Eur. Phys. J. C 72 (2012) 1847 [arXiv:1110.3726] [INSPIRE].

[23] CMS collaboration, Phenomenological MSSM interpretation of the CMS 2011 5fb-1 results, CMS-PAS-SUS-12-030.

[24] A. Fowlie, K. Kowalska, L. Roszkowski, E.M. Sessolo and Y.-L.S. Tsai, Dark matter and collider signatures of the MSSM, Phys. Rev. D 88 (2013) 055012 [arXiv:1306.1567] [INSPIRE].

[25] D. Feldman, Z. Liu and P. Nath, The Landscape of Sparticle Mass Hierarchies and Their Signature Space at the LHC, Phys. Rev. Lett. 99 (2007) 251802 [Erratum ibid. 100 (2008) 069902] [arXiv: 0707.1873] [INSPIRE].

[26] D. Feldman, Z. Liu and P. Nath, Sparticles at the LHC, JHEP 04 (2008) 054 [arXiv:0802.4085] [INSPIRE].

[27] J. Alwall, P. Schuster and N. Toro, Simplified Models for a First Characterization of New Physics at the LHC, Phys. Rev. D 79 (2009) 075020 [arXiv: 0810. 3921] [InSPIRE].

[28] LHC New Physics Working Group collaboration, D. Alves et al., Simplified Models for LHC New Physics Searches, J. Phys. G 39 (2012) 105005 [arXiv:1105.2838] [InSPIRE].

[29] N. Arkani-Hamed, G.L. Kane, J. Thaler and L.-T. Wang, Supersymmetry and the LHC inverse problem, JHEP 08 (2006) 070 [hep-ph/0512190] [INSPIRE].

[30] C.F. Berger, J.S. Gainer, J.L. Hewett, B. Lillie and T.G. Rizzo, Supersymmetry, the ILC and the LHC inverse problem, Phys. Lett. B 677 (2009) 48 [arXiv:0711.1374] [InSPIRE].

[31] B. Altunkaynak, M. Holmes and B.D. Nelson, Solving the LHC Inverse Problem with Dark Matter Observations, JHEP 10 (2008) 013 [arXiv:0804.2899] [INSPIRE].

[32] J.-L. Kneur and N. Sahoury, Bottom-Up Reconstruction Scenarios for (un)constrained MSSM Parameters at the LHC, Phys. Rev. D 79 (2009) 075010 [arXiv:0808.0144] [InSPIRE].

[33] C. Balázs and D. Kahawala, Stochastic resolution of the LHC inverse problem, AIP Conf. Proc. 1200 (2010) 482 [arXiv:0904.0128] [INSPIRE].

[34] P. Konar, K.T. Matchev, M. Park and G.K. Sarangi, How to look for supersymmetry under the lamppost at the LHC, Phys. Rev. Lett. 105 (2010) 221801 [arXiv:1008.2483] [INSPIRE].

[35] D.S.M. Alves, E. Izaguirre and J.G. Wacker, Where the Sidewalk Ends: Jets and Missing Energy Search Strategies for the 7 TeV LHC, JHEP 10 (2011) 012 [arXiv:1102.5338] [INSPIRE].

[36] T.J. LeCompte and S.P. Martin, Large Hadron Collider reach for supersymmetric models with compressed mass spectra, Phys. Rev. D 84 (2011) 015004 [arXiv:1105.4304] [INSPIRE].

[37] D.S.M. Alves, M.R. Buckley, P.J. Fox, J.D. Lykken and C.-T. Yu, Stops and $\mathbb{E}_{T}$ : The shape of things to come, Phys. Rev. D 87 (2013) 035016 [arXiv:1205.5805] [InSPIRE].

[38] H.K. Dreiner, F. Staub, A. Vicente and W. Porod, General MSSM signatures at the LHC with and without R-parity, Phys. Rev. D 86 (2012) 035021 [arXiv: 1205.0557] [INSPIRE].

[39] H.K. Dreiner, F. Staub and A. Vicente, General NMSSM signatures at the LHC, Phys. Rev. D 87 (2013) 035009 [arXiv: 1211.6987] [INSPIRE].

[40] J. Gainer, K. Matchev and M. Park, in preparation. 
[41] D.M. Pierce, J.A. Bagger, K.T. Matchev and R.-j. Zhang, Precision corrections in the minimal supersymmetric standard model, Nucl. Phys. B 491 (1997) 3 [hep-ph/9606211] [INSPIRE].

[42] K. Desch, J. Kalinowski, G.A. Moortgat-Pick, M.M. Nojiri and G. Polesello, SUSY parameter determination in combined analyses at LHC/LC, JHEP 02 (2004) 035 [hep-ph/0312069] [INSPIRE].

[43] R. Lafaye, T. Plehn and D. Zerwas, SFITTER: SUSY parameter analysis at LHC and $L C$, hep-ph/0404282 [INSPIRE].

[44] P. Bechtle, K. Desch and P. Wienemann, Fittino, a program for determining MSSM parameters from collider observables using an iterative method, Comput. Phys. Commun. 174 (2006) 47 [hep-ph/0412012] [INSPIRE].

[45] C. Boehm, P.S.B. Dev, A. Mazumdar and E. Pukartas, Naturalness of Light Neutralino Dark Matter in pMSSM after LHC, XENON100 and Planck Data, JHEP 06 (2013) 113 [arXiv:1303.5386] [INSPIRE].

[46] A.H. Chamseddine, R.L. Arnowitt and P. Nath, Locally Supersymmetric Grand Unification, Phys. Rev. Lett. 49 (1982) 970 [InSPIRE].

[47] R. Barbieri, S. Ferrara and C.A. Savoy, Gauge Models with Spontaneously Broken Local Supersymmetry, Phys. Lett. B 119 (1982) 343 [InSPIRE].

[48] N. Ohta, Grand unified theories based on local supersymmetry, Prog. Theor. Phys. 70 (1983) 542 [INSPIRE].

[49] L.J. Hall, J.D. Lykken and S. Weinberg, Supergravity as the Messenger of Supersymmetry Breaking, Phys. Rev. D 27 (1983) 2359 [INSPIRE].

[50] N. Polonsky and A. Pomarol, GUT effects in the soft supersymmetry breaking terms, Phys. Rev. Lett. 73 (1994) 2292 [hep-ph/9406224] [INSPIRE].

[51] N. Polonsky and A. Pomarol, Nonuniversal GUT corrections to the soft terms and their implications in supergravity models, Phys. Rev. D 51 (1995) 6532 [hep-ph/9410231] [INSPIRE].

[52] C.F. Kolda and S.P. Martin, Low-energy supersymmetry with D term contributions to scalar masses, Phys. Rev. D 53 (1996) 3871 [hep-ph/9503445] [INSPIRE].

[53] G. Anderson, C.H. Chen, J.F. Gunion, J.D. Lykken, T. Moroi and Y. Yamada, Motivations for and implications of nonuniversal GUT scale boundary conditions for soft SUSY breaking parameters, eConf C 960625 (1996) SUP107 [hep-ph/9609457] [INSPIRE].

[54] G. Anderson, H. Baer, C.-h. Chen and X. Tata, The Reach of Fermilab Tevatron upgrades for SU(5) supergravity models with nonuniversal gaugino masses, Phys. Rev. D 61 (2000) 095005 [hep-ph/9903370] [INSPIRE].

[55] J.L. Feng, M.E. Peskin, H. Murayama and X.R. Tata, Testing supersymmetry at the next linear collider, Phys. Rev. D 52 (1995) 1418 [hep-ph/9502260] [INSPIRE].

[56] P. Bechtle et al., Constrained Supersymmetry after two years of LHC data: a global view with Fittino, JHEP 06 (2012) 098 [arXiv: 1204.4199] [INSPIRE].

[57] O. Buchmueller et al., The CMSSM and NUHM1 in Light of 7 TeV LHC, Bs $\rightarrow \mu^{+} \mu^{-}$and XENON100 Data, Eur. Phys. J. C 72 (2012) 2243 [arXiv:1207.7315] [InSPIRE]. 
[58] C. Strege, G. Bertone, F. Feroz, M. Fornasa, R. Ruiz de Austri and R. Trotta, Global Fits of the CMSSM and NUHM including the LHC Higgs discovery and new XENON100 constraints, JCAP 04 (2013) 013 [arXiv:1212.2636] [INSPIRE].

[59] G.A. Blair, W. Porod and P.M. Zerwas, Reconstructing supersymmetric theories at high-energy scales, Phys. Rev. D 63 (2001) 017703 [hep-ph/0007107] [INSPIRE].

[60] G.A. Blair, W. Porod and P.M. Zerwas, The reconstruction of supersymmetric theories at high-energy scales, Eur. Phys. J. C 27 (2003) 263 [hep-ph/0210058] [INSPIRE].

[61] S.P. Martin and P. Ramond, Sparticle Spectrum Constraints, Phys. Rev. D 48 (1993) 5365 [hep-ph/9306314] [INSPIRE].

[62] H.C. Cheng and L.J. Hall, Squark and slepton mass relations in grand unified theories, Phys. Rev. D 51 (1995) 5289 [hep-ph/9411276] [INSPIRE].

[63] Y. Yamada, Radiative corrections to sfermion mass splittings, Phys. Rev. D 54 (1996) 1150 [hep-ph/9602279] [INSPIRE].

[64] A. Strumia, Distinguishing gauge mediated from unified supergravity spectra, Phys. Lett. B 409 (1997) 213 [hep-ph/9705306] [INSPIRE].

[65] K.T. Matchev, Precision corrections and supersymmetric unification, Ph.D. thesis, The Johns Hopkins University (1997), UMI-98-21162.

[66] K. Huitu, J. Laamanen and P.N. Pandita, Constraints on sparticle spectrum in different supersymmetry breaking models, Pramana 62 (2004) 663 [hep-ph/0303067] [INSPIRE].

[67] B. Ananthanarayan and P.N. Pandita, Squark and slepton masses as probes of supersymmetric SO(10) unification, Mod. Phys. Lett. A 19 (2004) 467 [hep-ph/0312361] [INSPIRE].

[68] D.A. Demir, Renormalization group invariants in the MSSM and its extensions, JHEP 11 (2005) 003 [hep-ph/0408043] [INSPIRE].

[69] B. Ananthanarayan and P.N. Pandita, Probing $\mathrm{SO}(10)$ symmetry breaking patterns through sfermion mass relations, Int. J. Mod. Phys. A 20 (2005) 4241 [hep-ph/0412125] [InSPIRE].

[70] Y. Kawamura and T. Kinami, More about superparticle sum rules in grand unified theories, Int. J. Mod. Phys. A 22 (2007) 4617 [arXiv:0705.1014] [InSPIRE].

[71] B. Ananthanarayan and P.N. Pandita, Sparticle Mass Spectrum in Grand Unified Theories, Int. J. Mod. Phys. A 22 (2007) 3229 [arXiv:0706.2560] [InSPIRE].

[72] S. Bhattacharya, A. Datta and B. Mukhopadhyaya, Non-universal gaugino masses: A signal-based analysis for the Large Hadron Collider, JHEP 10 (2007) 080 [arXiv:0708.2427] [INSPIRE].

[73] S. Bhattacharya, A. Datta and B. Mukhopadhyaya, Non-universal scalar masses: A signal-based analysis for the Large Hadron Collider, Phys. Rev. D 78 (2008) 035011 [arXiv: 0804.4051] [INSPIRE].

[74] M. Blanke, D. Curtin and M. Perelstein, SUSY-Yukawa Sum Rule at the LHC, Phys. Rev. D 82 (2010) 035020 [arXiv: 1004.5350] [INSPIRE].

[75] M. Carena, P. Draper, N.R. Shah and C.E.M. Wagner, Determining the Structure of Supersymmetry-Breaking with Renormalization Group Invariants, Phys. Rev. D 82 (2010) 075005 [arXiv: 1006 .4363] [INSPIRE]. 
[76] J. Jaeckel, V.V. Khoze and C. Wymant, Mass Sum Rules and the Role of the Messenger Scale in General Gauge Mediation, JHEP 04 (2011) 126 [arXiv:1102.1589] [INSPIRE].

[77] M. Carena, J. Lykken, S. Sekmen, N.R. Shah and C.E.M. Wagner, The pMSSM Interpretation of LHC Results Using Rernormalization Group Invariants, Phys. Rev. D 86 (2012) 075025 [arXiv: 1205.5903] [INSPIRE].

[78] D.J. Miller, A.P. Morais and P.N. Pandita, Constraining Grand Unification using first and second generation sfermions, Phys. Rev. D 87 (2013) 015007 [arXiv: 1208.5906] [INSPIRE].

[79] P. Ramond, Journeys beyond the standard model, Reading, Mass., Perseus Books, (1999).

[80] V. Berezinsky, A. Bottino, J.R. Ellis, N. Fornengo, G. Mignola and S. Scopel, Neutralino dark matter in supersymmetric models with nonuniversal scalar mass terms, Astropart. Phys. 5 (1996) 1 [hep-ph/9508249] [INSPIRE].

[81] P. Nath and R.L. Arnowitt, Nonuniversal soft SUSY breaking and dark matter, Phys. Rev. D 56 (1997) 2820 [hep-ph/9701301] [INSPIRE].

[82] http://www.hss.caltech.edu/ kcb/Notes/Alternative.pdf.

[83] J.L. Feng, A. Rajaraman and B.T. Smith, Minimal supergravity with m(0)**2 ; 0, Phys. Rev. D 74 (2006) 015013 [hep-ph/0512172] [INSPIRE].

[84] http://www.python.org. 